\title{
LONG-TERM TeV AND X-RAY OBSERVATIONS OF THE GAMMA-RAY BINARY HESS J0632+057
}

E. Aliu ${ }^{1}$, S. Archambault ${ }^{2}$, T. Aune ${ }^{3}$, B. Behera ${ }^{4}$, M. Beilicke ${ }^{5}$, W. Benbow ${ }^{6}$, K. Berger $^{7}$, R. Bird ${ }^{8}$, A. Bouvier ${ }^{9}$,

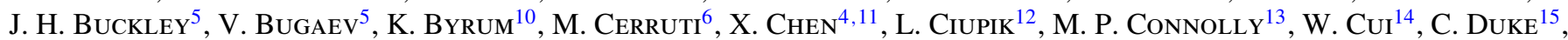
J. Dumm ${ }^{16}$, M. Errando ${ }^{1}$, A. Falcone ${ }^{17}$, S. Federici ${ }^{4}, 11$, Q. Feng ${ }^{14}$, J. P. Finley ${ }^{14}$, P. Fortin ${ }^{6}$, L. Fortson ${ }^{16}$, A. Furniss ${ }^{9}$,

N. Galante ${ }^{6}$, G. H. Gillanders ${ }^{13}$, S. Griffin ${ }^{2}$, S. T. Griffiths ${ }^{18}$, J. Grube ${ }^{12}$, G. GyuK ${ }^{12}$, D. Hanna ${ }^{2}$, J. Holder ${ }^{7}$,

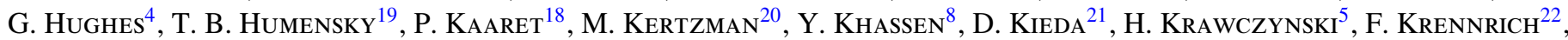
M. J. Lang ${ }^{13}$, A. S. Madhavan ${ }^{22}$, G. Maier ${ }^{4}$, P. Majumdar ${ }^{3,23}$, A. McCann ${ }^{24}$, P. Moriarty ${ }^{25}$, R. Mukherjee ${ }^{1}$, D. Nieto ${ }^{19}$, A. O'Faoláin de Bhróithe ${ }^{8}$, R. A. Ong ${ }^{3}$, A. N. Otte ${ }^{26}$, N. Park ${ }^{27}$, J. S. Perkins ${ }^{28}$, M. Pohl ${ }^{4,11}$, A. PopKow ${ }^{3}$, H. ProkOPH ${ }^{4}$,

J. QuinN ${ }^{8}$, K. Ragan ${ }^{2}$, J. Rajotte ${ }^{2}$, L. C. Reyes ${ }^{29}$, P. T. Reynolds ${ }^{30}$, G. T. Richards ${ }^{26}$, E. Roache ${ }^{6}$, J. Rousselle ${ }^{3}$, G. H. Sembroski ${ }^{14}$, F. Sheidaei ${ }^{21}$, C. Skole ${ }^{4}$, A. W. Smith ${ }^{21}$, D. StaszaK ${ }^{2}$, M. Stroh ${ }^{17}$, I. Telezhinsky ${ }^{4}, 11$, M. Theiling ${ }^{14}$,

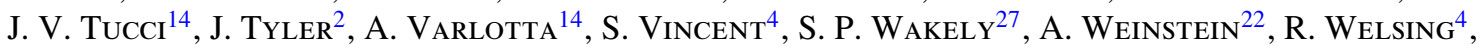

D. A. Williams ${ }^{9}$, A. ZAJCZYK ${ }^{5}$, B. ZitZeR ${ }^{10}$

(THE VERITAS COLlaboration)

A. Abramowski ${ }^{31}$, F. Aharonian ${ }^{32,33,34}$, F. Ait Benkhali ${ }^{32}$, A. G. Akhperjanian ${ }^{33,35}$, E. Angüner ${ }^{36}$, G. Anton Ant $^{37}$,

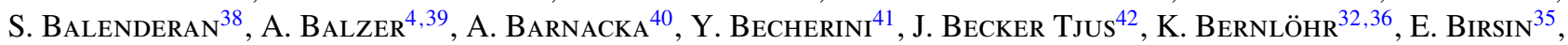
E. Bissaldi ${ }^{43}$, J. Biteau ${ }^{44}$, M. Böttcher ${ }^{45}$, C. Boisson ${ }^{46}$, J. Bolmont ${ }^{47}$, P. Bordas $^{48}$, J. Brucker ${ }^{37}$, F. Brun ${ }^{32}$, P. Brun ${ }^{49}$, T. Bulik ${ }^{50}$, S. Carrigan ${ }^{32}$, S. Casanova ${ }^{32,45}$, M. Cerrutit ${ }^{46,66}$, P. M. Chadwick ${ }^{38}$, R. Chalme-Calvet ${ }^{47}$, R. C. G. Chaves ${ }^{32,49}$, A. Cheesebrough ${ }^{38}$, M. Chrétien $^{47}$, S. Colafrancesco ${ }^{51}$, G. Cologna ${ }^{41}$, J. Conrad ${ }^{52}$, C. Couturier ${ }^{47}$, M. Dalton $^{53}$,

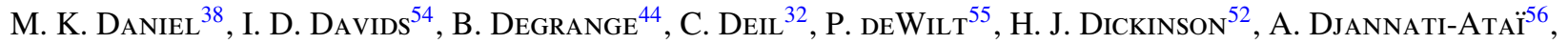
W. Domainko ${ }^{32}$, L. O'C. Drury ${ }^{33}$, G. Dubus ${ }^{57}$, K. Dutson ${ }^{58}$, J. Dyks ${ }^{40}$, M. Dyrda ${ }^{59}$, T. Edwards ${ }^{32}$, K. EgBerts ${ }^{43}$, P. $^{\text {Eger }}{ }^{32}$, P. Espigat ${ }^{56}$, C. Farnier ${ }^{52}$, S. Fegan ${ }^{44}$, F. Feinstein ${ }^{60}$, M. V. Fernandes ${ }^{31}$, D. Fernandez ${ }^{60}$, A. Fiasson ${ }^{61}$, G. Fontaine ${ }^{44}$, A. Förster ${ }^{32}$, M. Füssling ${ }^{39}$, M. Gajdus ${ }^{36}$, Y. A. Gallant ${ }^{32}$, T. Garrigoux ${ }^{47}$, G. Giavitto ${ }^{4}$, B. Giebels ${ }^{44}$, J. F. Glicenstein ${ }^{49}$,

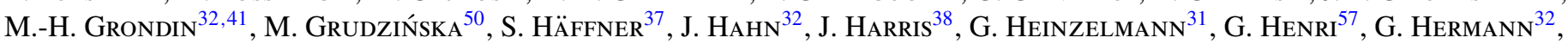

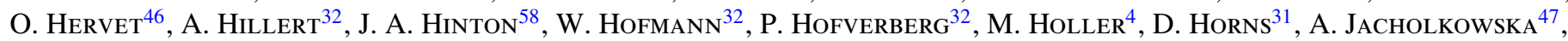

C. JAHN ${ }^{37}$, M. JAMROZY ${ }^{62}$, M. JANIAK ${ }^{40}$, F. JANKOWSKY ${ }^{41}$, I. JUNG ${ }^{37}$, M. A. KASTENDIECK ${ }^{31}$, K. KATARZYŃSKI ${ }^{63}$, U. KATZ ${ }^{37}$,

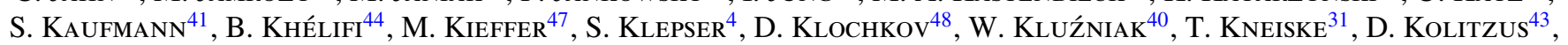
Nu. Komin ${ }^{61}$, K. Kosack ${ }^{49}$, S. Krakau ${ }^{42}$, F. Krayzel ${ }^{61}$, P. P. KrüGer ${ }^{32,45}$, H. LafFon ${ }^{53}$, G. Lamanna ${ }^{61}$, J. Lefaucheur ${ }^{56}$, A. Lemière ${ }^{56}$, M. Lemoine-Goumard ${ }^{53}$, J.-P. Lenain ${ }^{47}$, D. LenNARZ ${ }^{32}$, T. LOHSE $^{36}$, A. Lopatin ${ }^{37}$, C.-C. LU ${ }^{32}$, V. MARANDON ${ }^{32}$, A. Marcowith ${ }^{32}$, R. Marx ${ }^{32}$, G. Maurin ${ }^{61}$, N. Maxted ${ }^{55}$, M. MaYer ${ }^{39}$, T. J. L. McComb ${ }^{38}$, J. Méhault ${ }^{34,35}$, U. Menzler ${ }^{42}$, M. Meyer ${ }^{31}$, R. Moderski ${ }^{40}$, M. Mohamed ${ }^{41}$, E. Moulin ${ }^{49}$, T. Murach ${ }^{36}$, C. L. Naumann ${ }^{47}$, M. De Naurois ${ }^{44}$, J. Niemiec $^{60}$,

S. J. Nolan ${ }^{38}$, L. OAKes ${ }^{36}$, S. Ohi ${ }^{58,64}$, E. De OÑa Wilhelmi ${ }^{32}$, B. OPITZ ${ }^{31}$, M. Ostrowski ${ }^{64}$, I. Oya ${ }^{36}$, M. Panter ${ }^{32}$,

R. D. Parsons ${ }^{32}$, M. Paz Arribas ${ }^{36}$, N. W. Pekeur ${ }^{45}$, G. Pelletier ${ }^{57}$, J. Perez ${ }^{43}$, P.-O. Petrucci ${ }^{57}$, B. Peyaud ${ }^{49}$, S. Pita ${ }^{56}$, H. Poon ${ }^{60}$, G. PÜHLhofer ${ }^{48}$, M. Punch ${ }^{56}$, A. Quirrenbach ${ }^{41}$, S. RaAB ${ }^{37}$, M. RAuE $^{31}$, A. Reimer ${ }^{43}$, O. Reimer ${ }^{43}$, M. Renaud ${ }^{60}$,

R. DE los Reyes ${ }^{32}$, F. Rieger ${ }^{32}$, L. Rob ${ }^{65}$, C. Romoli ${ }^{33}$, S. Rosier-Lees ${ }^{33}$, G. Rowell ${ }^{55}$, B. Rudak ${ }^{40}$, C. B. Rulten ${ }^{46}$,

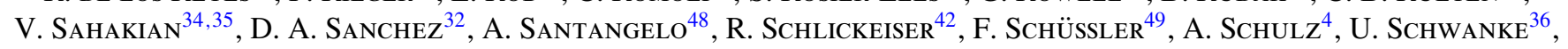

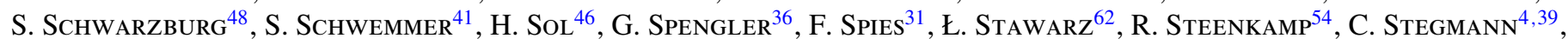

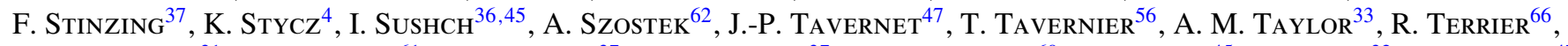
M. Tluczykont ${ }^{31}$, C. Trichard ${ }^{61}$, K. Valerius $^{37}$, C. van Eldik ${ }^{37}$, G. Vasileiadis ${ }^{60}$, C. Venter $^{45}$, A. Viana VI2 $^{32}$ P. Vincent $^{47}$,

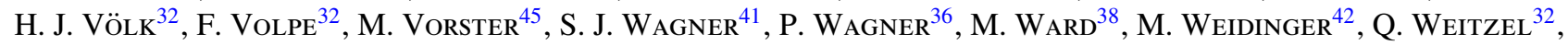

R. White ${ }^{58}$, A. Wierzcholska ${ }^{62}$, P. WillmanN ${ }^{37}$, A. Wörnlein ${ }^{37}$, D. WOUters ${ }^{49}$, M. Zacharias ${ }^{42}$, A. ZajCZYK ${ }^{40,60}$, A. A. ZdZiarski ${ }^{40}$, A. ZeCH ${ }^{46}$, H.-S. ZeChlin ${ }^{31}$

(H.E.S.S. Collaboration)

${ }^{1}$ Department of Physics and Astronomy, Barnard College, Columbia University, New York, NY 10027, USA

2 Physics Department, McGill University, Montreal, QC H3A 2T8, Canada

${ }^{3}$ Department of Physics and Astronomy, University of California, Los Angeles, CA 90095, USA

${ }^{4}$ DESY, Platanenallee 6, D-15738 Zeuthen, Germany; gernot.maier@ desy.de

${ }^{5}$ Department of Physics, Washington University, St. Louis, MO 63130, USA

${ }^{6}$ Fred Lawrence Whipple Observatory, Harvard-Smithsonian Center for Astrophysics, Amado, AZ 85645, USA

${ }^{7}$ Department of Physics and Astronomy and the Bartol Research Institute, University of Delaware, Newark, DE 19716, USA

${ }^{8}$ School of Physics, University College Dublin, Belfield, Dublin 4, Ireland

${ }^{9}$ Santa Cruz Institute for Particle Physics and Department of Physics, University of California, Santa Cruz, CA 95064, USA

${ }^{10}$ Argonne National Laboratory, 9700 S. Cass Avenue, Argonne, IL 60439, USA

${ }^{11}$ Institute of Physics and Astronomy, University of Potsdam, D-14476 Potsdam-Golm, Germany

12 Astronomy Department, Adler Planetarium and Astronomy Museum, Chicago, IL 60605, USA

${ }^{13}$ School of Physics, National University of Ireland Galway, University Road, Galway, Ireland

${ }^{14}$ Department of Physics, Purdue University, West Lafayette, IN 47907, USA

${ }^{15}$ Department of Physics, Grinnell College, Grinnell, IA 50112-1690, USA

${ }^{16}$ School of Physics and Astronomy, University of Minnesota, Minneapolis, MN 55455, USA

${ }^{17}$ Department of Astronomy and Astrophysics, 525 Davey Lab, Pennsylvania State University, University Park, PA 16802, USA; afalcone@ astro.psu.edu 


\author{
${ }^{18}$ Department of Physics and Astronomy, University of Iowa, Van Allen Hall, Iowa City, IA 52242, USA \\ ${ }^{19}$ Physics Department, Columbia University, New York, NY 10027, USA \\ ${ }^{20}$ Department of Physics and Astronomy, DePauw University, Greencastle, IN 46135-0037, USA \\ ${ }^{21}$ Department of Physics and Astronomy, University of Utah, Salt Lake City, UT 84112, USA \\ 22 Department of Physics and Astronomy, Iowa State University, Ames, IA 50011, USA \\ ${ }^{23}$ Saha Institute of Nuclear Physics, Kolkata 700064, India \\ ${ }^{24}$ Kavli Institute for Cosmological Physics, University of Chicago, Chicago, IL 60637, USA \\ ${ }^{25}$ Department of Life and Physical Sciences, Galway-Mayo Institute of Technology, Dublin Road, Galway, Ireland \\ ${ }^{26}$ School of Physics and Center for Relativistic Astrophysics, Georgia Institute of Technology, 837 State Street NW, Atlanta, GA 30332-0430, USA \\ ${ }^{27}$ Enrico Fermi Institute, University of Chicago, Chicago, IL 60637, USA \\ ${ }^{28}$ NASA Goddard Space Flight Center, Code 661, Greenbelt, MD 20771, USA \\ ${ }^{29}$ Physics Department, California Polytechnic State University, San Luis Obispo, CA 94307, USA \\ ${ }^{30}$ Department of Applied Physics and Instrumentation, Cork Institute of Technology, Bishopstown, Cork, Ireland \\ ${ }^{31}$ Institut für Experimentalphysik, Universität Hamburg, Luruper Chaussee 149, D-22761 Hamburg, Germany \\ 32 Max-Planck-Institut für Kernphysik, P.O. Box 103980, D-69029 Heidelberg, Germany \\ ${ }^{33}$ Dublin Institute for Advanced Studies, 31 Fitzwilliam Place, Dublin 2, Ireland \\ ${ }^{34}$ National Academy of Sciences of the Republic of Armenia, Yerevan, Armenia \\ ${ }^{35}$ Yerevan Physics Institute, 2 Alikhanian Brothers St., 375036 Yerevan, Armenia \\ ${ }^{36}$ Institut für Physik, Humboldt-Universität zu Berlin, Newtonstr. 15, D-12489 Berlin, Germany \\ ${ }^{37}$ Universität Erlangen-Nürnberg, Physikalisches Institut, Erwin-Rommel-Str. 1, D-91058 Erlangen, Germany \\ ${ }^{38}$ Department of Physics, University of Durham, South Road, Durham DH1 3LE, UK \\ ${ }^{39}$ Institut für Physik und Astronomie, Universität Potsdam, Karl-Liebknecht-Strasse 24/25, D-14476 Potsdam, Germany \\ ${ }^{40}$ Nicolaus Copernicus Astronomical Center, ul. Bartycka 18, 00-716 Warsaw, Poland \\ ${ }^{41}$ Landessternwarte, Universität Heidelberg, Königstuhl, D-69117 Heidelberg, Germany \\ ${ }^{42}$ Institut für Theoretische Physik, Lehrstuhl IV: Weltraum und Astrophysik, Ruhr-Universität Bochum, D-44780 Bochum, Germany \\ ${ }^{43}$ Institut für Astro-und Teilchenphysik, Leopold-Franzens-Universität Innsbruck, A-6020 Innsbruck, Austria \\ ${ }^{44}$ Laboratoire Leprince-Ringuet, Ecole Polytechnique, CNRS/IN2P3, F-91128 Palaiseau, France \\ ${ }^{45}$ Unit for Space Physics, North-West University, Potchefstroom 2520, South Africa \\ ${ }^{46}$ LUTH, Observatoire de Paris, CNRS, Université Paris Diderot, 5 Place Jules Janssen, F-92190 Meudon, France \\ ${ }^{47}$ LPNHE, Université Pierre et Marie Curie Paris 6, Université Denis Diderot Paris 7, CNRS/IN2P3, \\ 4 Place Jussieu, F-75252 Paris Cedex 5, France \\ ${ }^{48}$ Institut für Astronomie und Astrophysik, Universität Tübingen, Sand 1, D-72076 Tübingen, Germany; pol.bordas@ uni-tuebingen.de \\ ${ }^{49}$ DSM/Irfu, CEA Saclay, F-91191 Gif-Sur-Yvette Cedex, France \\ ${ }^{50}$ Astronomical Observatory, The University of Warsaw, Al. Ujazdowskie 4, 00-478 Warsaw, Poland \\ ${ }^{51}$ School of Physics, University of the Witwatersrand, 1 Jan Smuts Avenue, Braamfontein, 2050 Johannesburg, South Africa \\ 52 Oskar Klein Centre, Department of Physics, Stockholm University, Albanova University Center, SE-10691 Stockholm, Sweden \\ ${ }^{53}$ Université Bordeaux 1, CNRS/IN2P3, Centre d'Études Nucléaires de Bordeaux Gradignan, F-33175 Gradignan, France \\ ${ }^{54}$ University of Namibia, Department of Physics, Private Bag 13301, Windhoek, Namibia \\ ${ }^{55}$ School of Chemistry \& Physics, University of Adelaide, Adelaide 5005, Australia \\ ${ }^{56}$ APC, AstroParticule et Cosmologie, Université Paris Diderot, CNRS/IN2P3, CEA/Irfu, Observatoire de Paris, Sorbonne Paris Cité, \\ 10, rue Alice Domon et Léonie Duquet, F-75205 Paris Cedex 13, France \\ ${ }^{57}$ UJF-Grenoble 1/CNRS-INSU, Institut de Planétologie et d'Astrophysique de Grenoble (IPAG) UMR 5274, Grenoble F-38041, France \\ ${ }^{58}$ Department of Physics and Astronomy, The University of Leicester, University Road, Leicester LE1 7RH, UK \\ 59 Instytut Fizyki Jạdrowej PAN, ul. Radzikowskiego 152, 31-342 Kraków, Poland \\ ${ }^{60}$ Laboratoire Univers et Particules de Montpellier, Université Montpellier 2, CNRS/IN2P3, CC 72, Place Eugène Bataillon, \\ F-34095 Montpellier Cedex 5, France \\ ${ }^{61}$ Laboratoire d'Annecy-le-Vieux de Physique des Particules, Université de Savoie, CNRS/IN2P3, F-74941 Annecy-le-Vieux, France \\ ${ }^{62}$ Obserwatorium Astronomiczne, Uniwersytet Jagielloński, ul. Orla 171, 30-244 Kraków, Poland \\ ${ }^{63}$ Toruń Centre for Astronomy, Nicolaus Copernicus University, ul. Gagarina 11, 87-100 Toruń, Poland \\ ${ }^{64}$ School of Physics \& Astronomy, University of Leeds, Leeds LS2 9JT, UK \\ ${ }^{65}$ Charles University, Faculty of Mathematics and Physics, Institute of Particle and Nuclear Physics, V Holešovičkách 2, \\ 18000 Prague 8, Czech Republic \\ Received 2013 September 19; accepted 2013 November 22; published 2013 December 20
}

\begin{abstract}
HESS J0632+057 is the only gamma-ray binary known so far whose position in the sky allows observations with ground-based observatories in both the northern and southern hemispheres. Here we report on long-term observations of HESS J0632+057 conducted with the Very Energetic Radiation Imaging Telescope Array System and High Energy Stereoscopic System Cherenkov telescopes and the X-ray satellite Swift, spanning a time range from 2004 to 2012 and covering most of the system's orbit. The very-high-energy (VHE) emission is found to be variable and is correlated with that at X-ray energies. An orbital period of $315_{-4}^{+6}$ days is derived from the X-ray data set, which is compatible with previous results, $P=(321 \pm 5)$ days. The VHE light curve shows a distinct maximum at orbital phases close to 0.3 , or about 100 days after periastron passage, which coincides with the periodic enhancement of the X-ray emission. Furthermore, the analysis of the $\mathrm{TeV}$ data shows for the first time a statistically significant $(>6.5 \sigma)$ detection at orbital phases $0.6-0.9$. The obtained gamma-ray and X-ray light curves and the correlation of the source emission at these two energy bands are discussed in the context of the recent ephemeris obtained for the system. Our results are compared to those reported for other gamma-ray binaries.
\end{abstract}

Key words: acceleration of particles - binaries: general - gamma rays: general(HESS J0632+057, VER J0633+057)

Online-only material: color figures 


\section{INTRODUCTION}

The very-high-energy (VHE; $E>100 \mathrm{GeV}$ ) gamma-ray source HESS J0632+057 is a new member of the elusive class of gamma-ray binaries (Aharonian et al. 2007; Hinton et al. 2009; Bongiorno et al. 2011). These objects are characterized by a peak in their broad-band spectral energy distribution (SED) at $\mathrm{MeV}-\mathrm{GeV}$ energies, displaying variable high-energy emission as well as extended non-thermal radio structures. All known gamma-ray binaries are high-mass X-ray binary systems, consisting of a compact object orbiting around a massive star of O or Be type. Besides HESS J0632+057, only three binaries are clearly identified as VHE gamma-ray sources: PSR B1259-63/LS 2883 (Aharonian et al. 2005a), LS 5039 (Aharonian et al. 2005b), and LS I +61 303 (Albert et al. 2006; Acciari et al. 2008). In addition, some evidence for $\mathrm{TeV}$ emission has been observed from Cygnus X-1 with the Major Atmospheric Gamma-ray Imaging Cherenkov telescope (MAGIC; Albert et al. 2007). Finally, the High Energy Stereoscopic System (H.E.S.S.) Collaboration recently reported the detection of a point-like source spatially coincident with the newly discovered GeV gamma-ray binary 1FGL J1018-5859 (Fermi-LAT Collaboration 2012; Abramowski et al. 2012), although no variability could be identified at $\mathrm{TeV}$ energies and the complex morphology of the gamma-ray excess does not yet allow an unequivocal association of the $\mathrm{GeV}$ and $\mathrm{TeV}$ sources.

Gamma-ray emission at VHE from HESS J0632+057 was discovered serendipitously during observations of the Monoceros region in 2004-2005 with H.E.S.S. (Aharonian et al. 2007). Based on the point-like VHE gamma-ray appearance, X-ray variability, and spectral properties of the source, Aharonian et al. (2007) and Hinton et al. (2009) suggested its identification as a new TeV binary system. HESS J0632+057, located in the direction of the edge of the star-forming region of the Rosette Nebula, was observed in the following years with the Very Energetic Radiation Imaging Telescope Array System (VERITAS), H.E.S.S., and MAGIC telescopes. In 2006-2009, no significant emission was detected from the system at energies above $1 \mathrm{TeV}$ with VERITAS (Acciari et al. 2009), suggesting its variability at VHE. In 2010 and 2011 clear gamma-ray signals consistent with the initial H.E.S.S. results were observed (Maier et al. 2011; Aleksić et al. 2012; Bordas \& Maier 2012), confirming the $\mathrm{TeV}$ variability. The picture became clearer with the measurement of flux modulations with an initial period determination of $(321 \pm 5)$ days from multi-year observations in the $0.3-10 \mathrm{keV}$ band with the Swift X-ray telescope (XRT; Bongiorno et al. 2011; Falcone et al. 2010). The periodic modulation has been recently confirmed by photometric measurements in the optical band (Casares et al. 2012). The X-ray observations firmly established the binary nature of HESS J0632+057.

The optical counterpart of HESS J0632+057 is the massive B-star MWC 148 (HD 259440=LS VI +05 11) at a distance of 1.1-1.7 kpc (Aragona et al. 2010). MWC 148 is positionally coincident with the hard-spectrum and variable X-ray source XMMU J063259.3+054801 (Hinton et al. 2009). At radio frequencies, weak emission was discovered at the position of HESS J0632+057 with GMRT and Very Large Array by Skilton et al. (2009). The radio source is variable, but about 10 times fainter than the flux measured at similar frequencies from other gamma-ray binaries (e.g., LS 5039; Moldón et al. 2012). Observations with the European VLBI Network (EVN) show pos-

\footnotetext{
${ }^{66}$ Now at Harvard-Smithsonian Center for Astrophysics, 60 Garden Street,
} Cambridge, MA 02138, USA. sible indications of an extended radio structure with a projected size of $\approx 75$ AU (Moldón et al. 2011). Searches for pulsed emission from the system with Chandra and XMM-Newton in X-rays yielded upper limits of $\sim 22 \%-48 \%$ (depending on the frequency and emission state probed) on the pulsed fraction at energies $0.3-10 \mathrm{keV}$ (Rea \& Torres 2011). A potential association of HESS J0632+057 with the unidentified GeV gamma-ray source 3EG J0632+0521 has been suggested by Aharonian et al. (2007), although the source is marked as "possibly extended or multiple sources" and "possibly source confused" in the third EGRET catalog(Hartman et al. 1999). No gamma-ray emission has been reported by the Fermi Large Area Telescope (LAT) Collaboration (Caliandro et al. 2013) at $\mathrm{MeV}-\mathrm{GeV}$ energies $\left(F_{100}<3.0 \times 10^{-8}\right.$ photon $\mathrm{cm}^{-2} \mathrm{~s}^{-1} 99 \%$ C.L. upper limit above $100 \mathrm{MeV}$ ).

The spectral type of MWC 148 is B0pe (Morgan et al. 1955), characterized by an optically thick equatorial disk. The disk inclination is uncertain with estimates ranging from $\geqslant 47^{\circ}$ to between $71^{\circ}$ and $90^{\circ}$ (Casares et al. 2012). Estimates of the physical parameters of the star have been reported, e.g., by Aragona et al. (2010) and Casares et al. (2012), who derive an effective temperature of $T_{\text {eff }} \approx 30,000 \mathrm{~K}$, a mass of 13-19 $M_{\odot}$, and a radius of about 6-10 $R_{\odot}$. Orbital parameters of the binary system have been obtained through spectroscopic measurements assuming an orbital period of 321 days derived from the X-ray measurements (Casares et al. 2012), including the orbit eccentricity $e=0.83 \pm 0.08$, phase of the periastron $\Phi_{0}=0.967 \pm 0.008$ (defining phase 0 arbitrarily at $T_{0}=$ MJD 54857), and an uncertain inclination of $i \approx 47^{\circ}-90^{\circ}$. The large uncertainties involved in this calculation lead to a broad range of masses allowed for the compact object $\left(M_{c} \approx 1.3-7.1 M_{\odot}\right)$. Its nature, neutron star or black hole, is therefore unclear.

The physical processes leading to particle acceleration and gamma-ray emission in binaries are under debate. Two major classes of models are usually invoked to explain their highenergy emission (see, e.g., Mirabel 2012). In the first one, acceleration of charged particles takes place in accretionpowered relativistic jets (so called microquasars; Taylor \& Gregory 1984; Mirabel \& Rodriguez 1994) and usually implies a black hole as compact object. In the second one, highenergy emission is produced by the ultra-relativistic wind of a rotation-powered energetic pulsar, either scattering directly off the photon field of the companion star or photons from a circumstellar disk (Ball \& Kirk 2000; van Soelen \& Meintjes 2011; Khangulyan et al. 2012), or accelerating particles in the region where the pulsar wind collides with the disk material or the wind of the massive companion (Maraschi \& Treves 1981; Dubus 2006a). Note that the shocked wind material could also be relativistic in this last case (Bogovalov et al. 2008; Dubus et al. 2010).

The interactions between the compact object, the massive star, and their winds and magnetic fields form a complicated environment, in which acceleration, radiation, and absorption processes take place (see, e.g., Dubus 2013). This complex nature of gamma-ray binaries may lead to the variety of emission patterns observed in these systems. In particular, two of the best studied gamma-ray binaries show emission that is modulated by their orbital period (PSR B1259-63, Aharonian et al. 2005a; LS 5039, Aharonian et al. 2005b), while in the case of the system LS I +61 303 the source displays both periodic and episodic variability (Albert et al. 2009; Acciari et al. 2011; see also Chernyakova et al. 2012). 
Below, long-term gamma-ray observations of HESS J0632+ 057 with the VERITAS and H.E.S.S. facilities are reported, and $\mathrm{X}$-ray observations with the Swift-XRT telescope. Section 2 describes the VHE and the X-ray data sets, including VHE observations taken up to 2012 February and Swift-XRT observations up to $2012 \mathrm{March}$. These results provide for the first time a wide coverage of the system's orbital phases. Section 3 is focused on the results obtained from the VHE and X-ray data analysis. These results are discussed in Section 4 in a multi-wavelength context, and compared to those obtained from other well-studied gamma-ray binaries.

\section{OBSERVATIONS}

VERITAS and H.E.S.S. are ground-based imaging atmospheric Cherenkov telescopes (IACTs) built to detect the faint flashes of Cherenkov light from air showers initiated in the atmosphere by high-energy gamma-ray photons. The instruments are very similar in their performance with effective areas of $\gtrsim 10^{5}$ $\mathrm{m}^{2}$ over an energy range from $\sim 100 \mathrm{GeV}$ to $30 \mathrm{TeV}$, energy resolution $\sim 15 \%-20 \%$, and angular resolution $\approx 0.1$. The high sensitivities of H.E.S.S. and VERITAS enable the detection of sources with a flux of $1 \%$ of the Crab Nebula in less than $30 \mathrm{hr}$ of observations.

The analysis of the VHE data from the two instruments follows similar initial steps, consisting of calibration, image cleaning, and second-moment parameterization of Cherenkov shower images (Hillas 1985), which provide the reconstruction of the shower direction, energy, and impact parameter using stereoscopic methods (see, e.g., Krawczynski et al. 2006). For the H.E.S.S. data analysis, a further fitting procedure is employed, for which the Hillas parameters are used as the starting point for a refined derivation of the shower parameters based on a log-likelihood comparison of the raw, uncleaned image with a pre-calculated shower model (de Naurois \& Rolland 2009). A shower event must be imaged by at least two out of four telescopes to be used in the VHE analysis of data from both instruments, and additional cuts on the shape of the event images and the direction of the primary particles are used to reject the far more numerous background events. Most of the VHE data were taken in wobble mode in both the VERITAS and H.E.S.S. data sets, wherein the source is positioned at an offset from the camera center of about 0.5 to allow for simultaneous and symmetric background regions to be used during the data-analysis procedure. All results presented here have been cross-checked with independent analysis chains.

\subsection{VERITAS VHE Gamma-Ray Observations}

The VERITAS observatory is an array of IACTs located at the Fred Lawrence Whipple Observatory in southern Arizona (1300 $\mathrm{m}$ above sea level, N31 $\left.40^{\prime} 30^{\prime \prime}, \mathrm{W} 110^{\circ} 57^{\prime} 08^{\prime \prime}\right)$. The mirror area of each telescope is $110 \mathrm{~m}^{2}$ and the total field of view $(\mathrm{FoV})$ of the instrument is 3.5 in diameter.

VERITAS observed the sky around HESS J0632+057 for a total of $162 \mathrm{hr}$ between 2006 December and 2012 January (see Table 1 for details). A total of $144 \mathrm{hr}$ of observations passed quality-selection criteria, which remove data taken during bad weather or with technical problems. The instrument went through several important changes during this period. The data from 2006 December were taken during the construction phase of VERITAS with three telescopes only. The array was completed in 2007 September with four telescopes in total. In 2009 September the array layout was improved by moving one telescope, leading to an improved sensitivity, which makes it possible to detect point-like sources with a flux of $1 \%$ of the Crab Nebula in less than $30 \mathrm{hr}$ of observations ( $1 \mathrm{hr}$ for $5 \%$ of the Crab Nebula flux). The corresponding values prior to 2009 are less than $50 \mathrm{hr}$ and $2 \mathrm{hr}$ for sources with $1 \%$ and $5 \%$ of the flux of the Crab Nebula respectively.

Observations with VERITAS are possible during dark sky and moderate moonlight conditions (moon illumination $<35 \%$ ). The elevated background light levels during moderate moonlight lead to a lower sensitivity to gamma rays near the low energy threshold of the instrument. Observations were performed in a zenith angle range of $26^{\circ}-40^{\circ}$. All VERITAS observations, with the exception of the observations in 2006 December-2007 January, were taken at a fixed offset of 0.5 in one of four directions. The energy threshold ${ }^{67}$ after analysis cuts, where a cut on the integrated charge per image of 500 digital counts ( $\approx 90$ photoelectrons) is applied, is $230 \mathrm{GeV}$ for the data set presented here (average elevation angle of $62^{\circ}$ ). For more details on the VERITAS instrument see, e.g., Acciari et al. (2008). The extraction region for photons from the putative gamma-ray source is defined by a 0.09 radius circle centered on the position of the X-ray source XMMU J063259.3+054801 (coincident with the star MWC 148; Hog et al. 1998). The background in the source region is estimated from the same FoV using the ring-background model with a ring size of 0.5 (mean radius) and a ring width of 0.1 (Berge et al. 2007). In order to reduce systematics in the background estimation, regions around stars with $B$-band magnitudes brighter than six are excluded from the background control regions.

\subsection{H.E.S.S. VHE Gamma-Ray Observations}

The H.E.S.S. observatory is located in the Khomas highland of Namibia (1800 $\mathrm{m}$ above sea level, S23 $3^{\circ} 16^{\prime} 18^{\prime \prime}$, E16 $30^{\circ} 00^{\prime \prime}$ ). The H.E.S.S. array consists of four $13 \mathrm{~m}$ diameter telescopes positioned in a square of side length $120 \mathrm{~m}$. Each telescope is equipped with a tessellated spherical mirror of $107 \mathrm{~m}^{2}$, focusing the Cherenkov flashes onto a camera that covers an FoV of about $5^{\circ}$ in diameter. For a detailed description of the system, see Aharonian et al. (2006) and references therein. No data from the H.E.S.S.-II array, which includes the addition of a central, $28 \mathrm{~m}$ diameter telescope, are included in this publication.

HESS J0632+057 was observed yearly with H.E.S.S. from 2004 until 2012. The FoV around the source was initially covered by deep observations of the Monoceros Loop supernova remnant/Rosette Nebula region, in the search for potential sources of VHE emission, including also two unidentified EGRET sources. After the discovery of HESS J0632+057 (Aharonian et al. 2007), further dedicated observations were obtained to better constrain its nature, in particular searching for $\mathrm{TeV}$ variability/periodicity following the non-detection of the source reported by the VERITAS Collaboration in 2006-2009 (Acciari et al. 2009). The total acceptance-corrected effective exposure time on HESS J0632+057, including both the initial discovery data set and the following pointed observations, consists of $53.5 \mathrm{hr}$ of data after standard quality selection cuts (Aharonian et al. 2006). Observations were performed over a large range of elevation angles $\left(32^{\circ}-62^{\circ}\right.$, with an average of 56.0). The data have been analyzed using the Model Analysis

\footnotetext{
67 The energy threshold is defined as the position of the peak of the differential energy spectrum (assuming a power law for the spectral shape; here $\Gamma=-2.5$ was assumed) of the source convolved with the effective area curve of the detector.
} 
Table 1

H.E.S.S. and VERITAS Analysis Results for Energies $E>1 \mathrm{TeV}$

\begin{tabular}{|c|c|c|c|c|c|c|c|c|c|}
\hline $\begin{array}{l}\text { MJD } \\
\text { Range }\end{array}$ & $\begin{array}{c}\text { Mean } \\
\text { Phase }^{\mathrm{a}}\end{array}$ & Observatory & $\begin{array}{l}\text { Observation } \\
\text { Time } \\
\text { (minutes) }\end{array}$ & $\begin{array}{l}\text { Mean } \\
\text { Elevation } \\
\quad(\text { deg })\end{array}$ & $\begin{array}{c}\text { On } \\
\text { Events }\end{array}$ & $\begin{array}{c}\text { Off } \\
\text { Events }\end{array}$ & $\alpha^{\mathrm{b}}$ & $\begin{array}{c}\text { Significance }^{c} \\
(\sigma) \\
\text { (Pre-trial) }\end{array}$ & $\begin{array}{c}\text { Flux } \\
\left(\text { Upper Flux Limit }^{\mathrm{d}}\right) \\
\left(10^{-13} \mathrm{~cm}^{-2} \mathrm{~s}^{-1}\right)\end{array}$ \\
\hline $54089-54125$ & 0.62 & VERITAS & 579 & 61.3 & 12 & 153 & 0.06 & 1.1 & $1.4 \pm 1.5(<6.1)$ \\
\hline $54830-54834$ & 0.92 & VERITAS & 561 & 62.6 & 7 & 180 & 0.05 & -0.9 & $-0.9 \pm 0.9(<2.3)$ \\
\hline $54856-54861$ & 0.01 & VERITAS & 721 & 61.9 & 12 & 197 & 0.05 & 0.4 & $0.4 \pm 1.0(<3.6)$ \\
\hline $55122-55133$ & 0.86 & VERITAS & 491 & 62.6 & 9 & 78 & 0.05 & 2.0 & $1.8 \pm 1.1(<4.5)$ \\
\hline $55259-55276$ & 0.30 & VERITAS & 309 & 59.5 & 18 & 55 & 0.05 & 5.6 & $8.2 \pm 2.3$ \\
\hline $55544-55564$ & 0.21 & VERITAS & 229 & 62.7 & 4 & 46 & 0.05 & 0.9 & $1.2 \pm 1.6(<7.1)$ \\
\hline $55571-55572$ & 0.27 & VERITAS & 140 & 61.7 & 4 & 23 & 0.05 & 1.9 & $3.4 \pm 2.5(<12.8)$ \\
\hline $55585-55599$ & 0.33 & VERITAS & 639 & 58.6 & 25 & 106 & 0.05 & 5.7 & $5.3 \pm 1.4$ \\
\hline $55600-55602$ & 0.36 & VERITAS & 541 & 58.9 & 27 & 104 & 0.05 & 6.2 & $6.9 \pm 1.7$ \\
\hline $55614-55622$ & 0.42 & VERITAS & 643 & 58.7 & 14 & 105 & 0.05 & 2.9 & $2.2 \pm 1.0(<5.5)$ \\
\hline $55624-55630$ & 0.45 & VERITAS & 342 & 57.8 & 5 & 77 & 0.05 & 0.4 & $0.4 \pm 1.2(<4.6)$ \\
\hline 55891-55901 & 0.30 & VERITAS & 454 & 61.0 & 21 & 90 & 0.05 & 5.2 & $6.2 \pm 1.8$ \\
\hline $55916-55920$ & 0.37 & VERITAS & 632 & 59.8 & 31 & 98 & 0.05 & 7.3 & $6.9 \pm 1.5$ \\
\hline $55921-55927$ & 0.39 & VERITAS & 419 & 62.4 & 8 & 67 & 0.05 & 1.9 & $1.9 \pm 1.2(<6.0)$ \\
\hline $55940-55949$ & 0.45 & VERITAS & 295 & 62.4 & 2 & 35 & 0.05 & 0.1 & $0.1 \pm 0.9(<3.8)$ \\
\hline 55951-55955 & 0.48 & VERITAS & 256 & 62.7 & 4 & 25 & 0.05 & 1.8 & $1.8 \pm 1.3(<6.8)$ \\
\hline $53087-53088$ & 0.38 & H.E.S.S. & 77.7 & 43.9 & 13 & 185 & 0.05 & 1.1 & $1.3 \pm 1.4(<4.2)$ \\
\hline $53353-53356$ & 0.23 & H.E.S.S. & 290.6 & 54.3 & 110 & 1209 & 0.05 & 6.0 & $4.0 \pm 0.8$ \\
\hline $53685-53716$ & 0.32 & H.E.S.S. & 324.7 & 53.4 & 113 & 1175 & 0.04 & 7.2 & $4.9 \pm 0.9$ \\
\hline $53823-53823$ & 0.71 & H.E.S.S. & 79.1 & 47.5 & 22 & 328 & 0.03 & 3.0 & $4.7 \pm 1.9$ \\
\hline $54117-54118$ & 0.65 & H.E.S.S. & 254.9 & 58.7 & 72 & 933 & 0.05 & 3.1 & $2.0 \pm 0.7$ \\
\hline $54169-54170$ & 0.81 & H.E.S.S. & 54.3 & 59.7 & 5 & 141 & 0.05 & -0.8 & $-0.9 \pm 0.09(<1.4)$ \\
\hline $54414-54426$ & 0.61 & H.E.S.S. & 156.4 & 59.5 & 50 & 538 & 0.05 & 3.7 & $3.3 \pm 1.0$ \\
\hline $54467-54475$ & 0.77 & H.E.S.S. & 217.1 & 58.4 & 69 & 644 & 0.05 & 5.2 & $3.9 \pm 0.9$ \\
\hline $54859-54910$ & 0.08 & H.E.S.S. & 161.6 & 52.1 & 26 & 368 & 0.05 & 1.5 & $1.3 \pm 0.09(<3.3)$ \\
\hline $55895-55898$ & 0.29 & H.E.S.S. & 230.7 & 59.4 & 87 & 885 & 0.05 & 5.2 & $3.6 \pm 0.8$ \\
\hline $55931-55951$ & 0.44 & H.E.S.S. & 233.2 & 52.5 & 53 & 673 & 0.05 & 2.9 & $2.1 \pm 0.8(<3.7)$ \\
\hline
\end{tabular}

Notes.

a Phases are calculated using an orbital period of $315 \mathrm{~d}$ and $\mathrm{MJD}_{0}=54857$.

${ }^{\mathrm{b}} \alpha$ denotes the ratio between the area used for the determination of on and off counts.

${ }^{c}$ Significances are calculated using Equation (17) from Li \& Ma (1983).

${ }^{\mathrm{d}}$ Errors on fluxes are $1 \sigma$ statistical uncertainties. Upper limits $(E>1 \mathrm{TeV})$ are given in brackets at $99 \%$ confidence level (after Rolke et al. 2001) for periods with a significance lower than $3 \sigma$.

technique (de Naurois \& Rolland 2009) and cross checked with a Hillas-based analysis, making use of an independent calibration procedure of the raw data, providing compatible results. The results presented here, using standard cuts where a cut of 60 photo-electrons on the intensity of the extensive air showers is applied, provide a mean energy threshold of $\sim 220 \mathrm{GeV}$ for the data set presented.

\subsection{Swift X-Ray Observations}

The Swift-XRT, which is sensitive in the $0.3-10 \mathrm{keV}$ band (Burrows et al. 2005), was used to monitor HESS J0632+057 during the time period from 2009 January 26 to 2012 February 15. The observations had typical durations of $\sim 4-5 \mathrm{ks}$. The temporal spacing between observations is typically $\sim 1$ week, although some time periods include several month-long time gaps due to observing constraints, whilst others include daily observations.

The Swift-XRT data were processed using the most recent versions of the standard Swift tools: Swift Software version 3.9 and HEASoft FTOOLS version 6.12 (Blackburn 1995). Observations were processed individually using xrtpipeline version 0.12.6. Hot and flickering pixels were removed using xrthotpix, and hot pixels were additionally removed by rejecting data where the XRT CCD temperature is $\geqslant-47^{\circ} \mathrm{C}$. Standard grade selections of $0-12$ were used for these Photon-Counting mode data.

Light curves were generated using xrtgrblc version 1.6. Circles were used to describe the source regions. The source count rate was always $<0.1$ counts $\mathrm{s}^{-1}$, so pile-up correction was not needed. Annuli with inner radii outside the radii of the source regions were used to describe the background regions for all data. The radii of the regions depend on the count rate in each temporal bin. Point-spread-function corrections and corrections for the relative sizes of the extraction regions were applied. For light-curve plotting, each observation results in one bin.

In order to calculate count-rate to flux conversion factors, spectral fits were generated using XSPEC version 12.7.1 (Arnaud 1996). Since the spectral shape may vary, we defined high, medium and low states based on the rate light curve, and binned data together within each subset to perform a spectral fit. The high state contains only data from the large peak (see Figure 3), approximately at phases $0.32-0.39$. The low state 


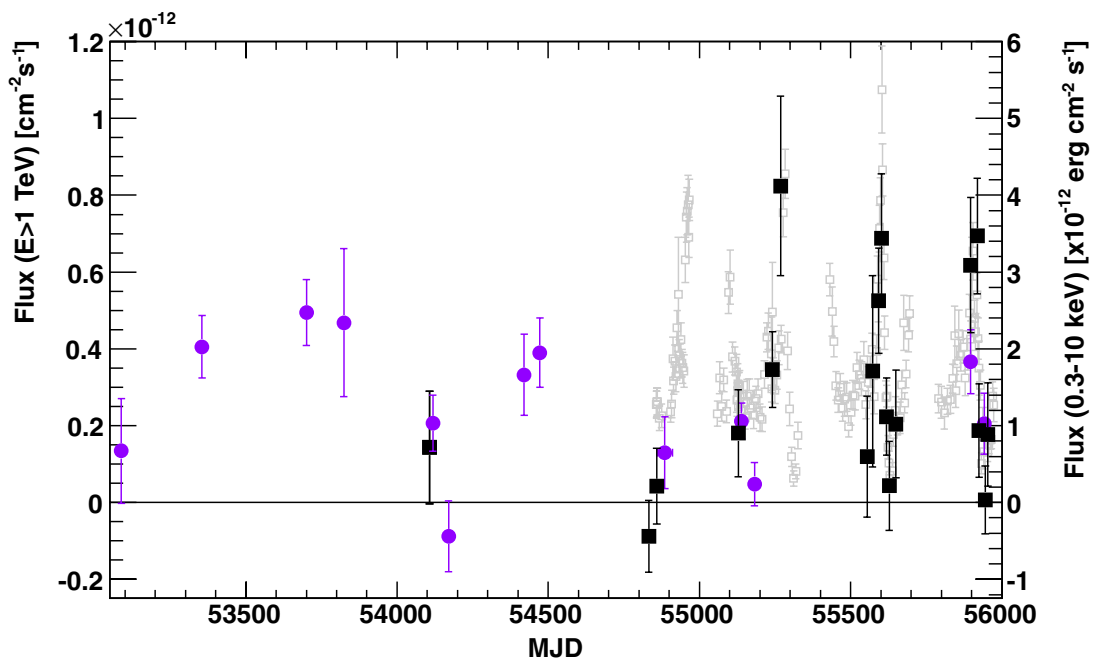

Figure 1. Long-term observations of HESS J0632+057 with H.E.S.S. (round purple markers) and VERITAS (square black markers) at energies $>1$ TeV and in X-rays with Swift-XRT (0.3-10 keV; open gray markers) from 2004 March to 2012 February.

(A color version of this figure is available in the online journal.)

Table 2

Outcome of the Spectral Analysis of the Swift X-ray Data

\begin{tabular}{lcccc}
\hline \hline Flux State & Phase Range & $\begin{array}{c}\text { Flux Normalization Constant }\left(\times 10^{-4}\right) \\
\left(\mathrm{cm}^{-2} \mathrm{~s}^{-1} \mathrm{keV}^{-1}\right)\end{array}$ & Photon Index & $\chi^{2} / N$ \\
\hline High & $0.32-0.39$ & $(4.25 \pm 0.24)$ & $1.46 \pm 0.06$ & $80 / 78$ \\
Medium & $\ldots$ & $(2.09 \pm 0.08)$ & $1.71 \pm 0.04$ & $224 / 168$ \\
Low & $0.42-0.48$ & $(0.50 \pm 0.01)$ & $1.19 \pm 0.16$ & $6 / 14$ \\
\hline
\end{tabular}

Notes. Parameters are derived from fitting an absorbed power law with a fixed absorption coefficient $N_{\mathrm{H}}=$ $3.81 \times 10^{21} \mathrm{~cm}^{-2}$. The spectral fit on medium flux states is added for illustration. Data for this fit are selected according to their absolute flux values and not in a specific phase range.

contains only data during the "dip" that immediately follows each large peak, roughly from phase $0.42-0.48$. The medium state data contains data from phase 0.6 to phase 0.32 (see Table 2). An absorbed power-law was used to fit the spectra from each of these three data subsets, using $\chi^{2}$ statistics and the $X$ Spec photoelectric model wabs to define absorption cross sections and abundances (Morrison \& McCammon 1983). For the combined data set, $N_{\mathrm{H}}$ was left as a free parameter and converged to $3.81(+0.29,-0.27) \times 10^{21} \mathrm{~cm}^{-2}$. For each of the data subsets, we fixed the absorption parameter, $N_{\mathrm{H}}$, to $3.81 \times 10^{21} \mathrm{~cm}^{-2}$. After performing a spectral fit of an absorbed power-law to each data subset, the unabsorbed flux was calculated to create three separate rate-to-flux conversion factors.

\section{RESULTS}

HESS J0632+057 was detected as a source of VHE gamma rays at a high confidence level by both observatories. The detection significance of the highly variable gamma-ray source derived from $144 \mathrm{hr}$ of VERITAS observations is $15.5 \sigma$, whereas the source is detected with H.E.S.S. at a significance of $13.6 \sigma$ in a total of $53.5 \mathrm{hr}$ of observations. Figure 1 summarizes these results by showing the long-term gammaray light curve for energies above $1 \mathrm{TeV}$ for all H.E.S.S. and VERITAS measurements from 2004 to 2012 (detailed results of these observations can be found in Table 1). All significances, fluxes, and spectral analyses are calculated using the X-ray source XMMU J063259.3+054801 position (Hinton et al. 2009). Integral fluxes above $1 \mathrm{TeV}$ are calculated assuming a spectral distribution following a power law with a photon index of -2.5 (see Figure 7 for the measured differential energy spectrum of HESS J0632+057).

The source has been found to be variable, as earlier measurements with H.E.S.S. and VERITAS suggested (Acciari et al. 2009). A calculation of the variability index $V$, a $\chi^{2}$-criterion described in detail in Abdo et al. (2010), results in $V=94.7$ for the combined H.E.S.S./VERITAS light curve with 30 flux points and the assumption of a systematic error on the flux estimation of each instrument of $20 \%$ (Aharonian et al. 2006). This means that the light curve is significantly different from a constant one at a confidence level of $1-3.6 \times 10^{-13}(7.1 \sigma)$. A search for variability patterns and an orbital period in the VHE data is unfortunately hampered by insufficient coverage and large gaps in the light curve. A much larger data set with denser sampling of the orbital period is needed to derive this from observations of HESS J0632+057.

The high-significance detection allows the position of the VHE source to be updated using both the VERITAS (from 2010 to 2012) and H.E.S.S. (from 2004 to 2012) data sets. The best fit position from the VERITAS subset is R.A. $=06^{\mathrm{h}} 33^{\mathrm{m}} 0.8 \pm 0.5_{\text {stat }}^{\mathrm{s}}$ and decl. $=+5^{\circ} 47^{\prime} 39^{\prime \prime} \pm 10^{\prime \prime}$ stat $(\mathrm{J} 2000$ coordinates $)$ with a source extension of the gamma-ray image compatible with the VERITAS gamma-ray point spread function. ${ }^{68}$ The systematic uncertainty in the position due to telescope pointing errors is below 50". This subset of VERITAS data, with observation dates after the optimization of the array layout, has been chosen to minimize systematic errors on the direction determination. The best-fit position for HESS J0632+057 resulting from the

68 The object is added to the VERITAS source catalog with the name VER J0633+057. 


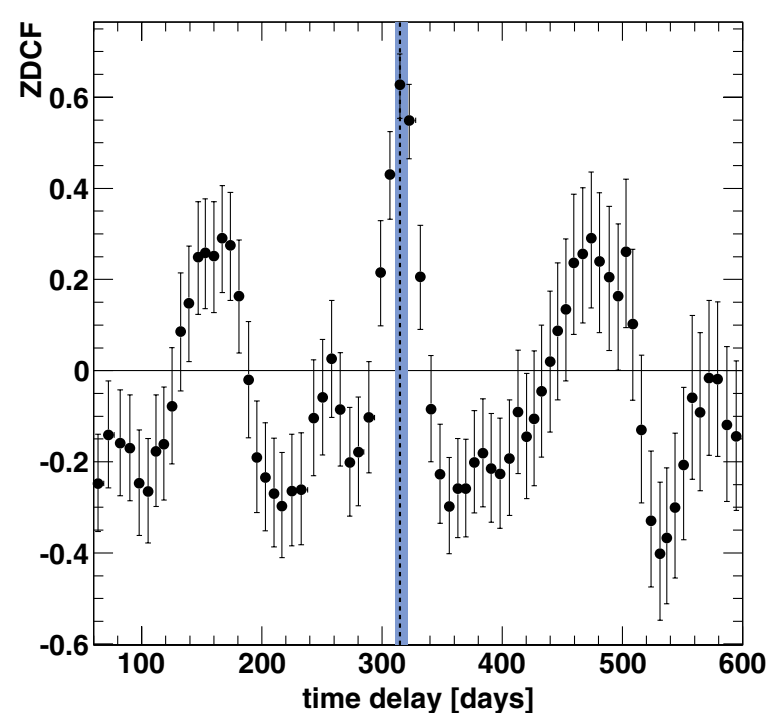

Figure 2. Z-transformed discrete autocorrelation function (Z-DCF) for the SwiftXRT light curve shown in Figure 1. The errors bars denote the $1 \sigma$ sampling errors resulting from a Monte Carlo-based error calculation as described in the text. The dashed line and the blue band indicate the most likely modulation period of $315_{-4}^{+6}$ days and the corresponding $68 \%$ fiducial interval.

(A color version of this figure is available in the online journal.)

new H.E.S.S. measurements is R.A. $=06^{\mathrm{h}} 32^{\mathrm{m}} 59^{\mathrm{s}} .4 \pm 1^{\mathrm{s}} \cdot 1_{\text {stat }}$ and decl. $=+5^{\circ} 47^{\prime} 20^{\prime \prime} \pm 16^{\prime \prime} 1_{\text {stat }}(\mathrm{J} 2000)$. The positional agreement between the updated VERITAS and H.E.S.S. position, the original H.E.S.S. detection (Aharonian et al. 2007) and the X-ray source XMMU J063259.3+054801, as well as its compatibility with a point-like source, have thus been confirmed.

The long-term X-ray light curve of XMMU J063259.3+ 054801, from 2009 January 26 to 2012 February 12, as measured with Swift-XRT is shown together with the VERITAS and H.E.S.S. measurements in Figure 1. The X-ray light curve is highly variable with several distinguishable features appearing periodically. The analysis reported here follows closely that in Bongiorno et al. (2011), but using one additional year of data. Z-transformed discrete correlation functions (Z-DCFs) are applied to determine the overall variability patterns in the $X$-ray light curve and the correlation between X-ray and gamma-ray emission (the number of data points in the gamma-ray light curve is not sufficient for an autocorrelation analysis). The Z-transformed discrete correlation functions (Alexander 1997) are based on the discrete correlation analysis developed by Edelson \& Krolik (1997), employing additionally equal population binning and Fisher's Z-transform, that transforms the correlation coefficient into an approximately normally distributed variable. This leads to a more robust estimation of the correlation coefficients. Errors on the Z-DCF coefficients are calculated in this analysis with a Monte Carlo-based approach using 10,000 simulated light curves with flux values randomly changed according to their measurement uncertainties and assuming them to be normally distributed. Time lags and their $68 \%$ fiducial intervals are calculated from the peak likelihood of the Z-DCF using Bayesian statistics. Z-DCF have been used in preference to Pearson's correlation coefficient as the latter does not take uncertainties on the flux values into account.

Figure 2 shows the results from the autocorrelation analysis of the X-ray light curve. Flux modulation with a period of $(321 \pm 5)$ days has been presented in Bongiorno et al. (2011), applying peak-fitting algorithms and Z-DCFs to a subset of

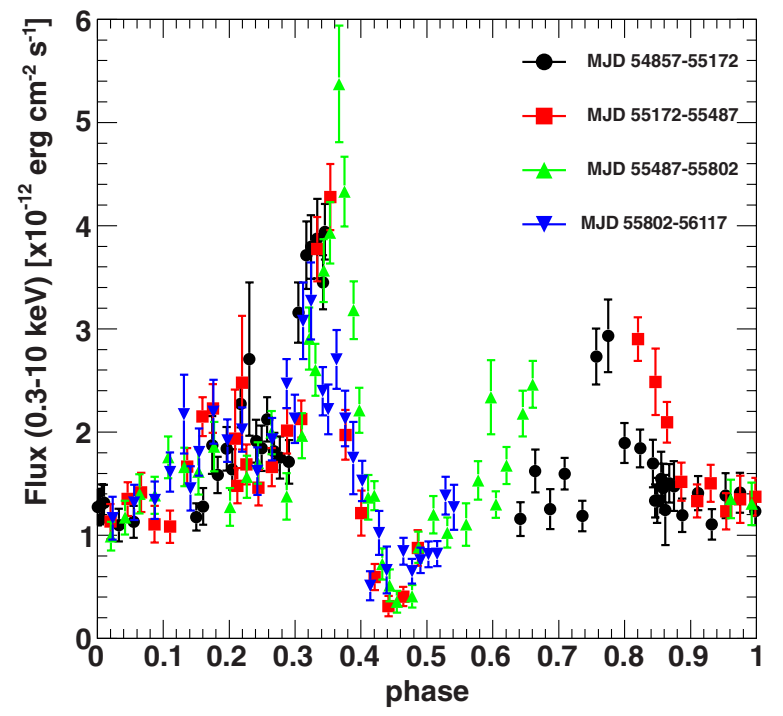

Figure 3. Phase-folded X-ray $(0.3-10 \mathrm{keV})$ light curve assuming an orbital period of 315 days. Different orbital cycles are indicated by markers and colors. Vertical error bars show $1 \sigma$ statistical uncertainties.

(A color version of this figure is available in the online journal.)

the Swift-XRT data presented in this paper. The larger data set available now (154 compared to 112 flux points used in Bongiorno et al. 2011) results in a compatible period of $315_{-4}^{+6}$ days. We use therefore in this paper the following phase definition: $\mathrm{MJD}_{0}=54857$ (arbitrarily set to the date of the first Swift observations) and period $P=315$ days. It should be noted that the orbital parameters as derived from Casares et al. (2012) remain approximately unchanged by this small change in orbital period (J. Casares 2012, private communication).

The phase-folded X-ray light curves as shown in Figure 3 illustrate the very regular emission pattern of HESS J0632+057 with a strong maximum at phases $\sim 0.35$, a marked dip at phases $\sim 0.45$ and an intermediate flux level at orbital phases $\sim 0.6-0.3$. There are also indications of a second maximum at phases $\sim 0.6-0.9$, with a flux level about half of that of the main peak at phases $\sim 0.35$. Apart from this very regular pattern, orbit-toorbit variability at X-ray energies is also visible, e.g., around the region of the emission maxima.

The gamma-ray light curve has been folded with the orbital period derived from the X-ray data. The uncertainty in the orbital period translates into a noticeable uncertainty in orbital phase, since the VHE observations presented here are taken over a period of $\sim 8 \mathrm{yr}$. In order to ensure that none of the conclusions presented in the following depends on the particular value of the orbital period, we present in Figure 4 the gammaray light curve folded by a period of 315 days, while in Figure 5 periods of 321 and 311 days have been applied. The phasefolded light curves reveal several important characteristics of the high-energy emission: a clear detection of the source in the phase range $0.2-0.4$, around the maximum of the X-ray light curve, with a flux of $2 \%-3 \%$ of that of the Crab Nebula; a first detection of a gamma-ray emission component at orbital phases in the range $0.6-0.9$, in which a secondary peak in the X-ray light curve is also observed; and a non-detected low state at all other orbital phases.

It should be noted that the H.E.S.S. data set leading to the detection of VHE emission in orbital phases 0.6-0.9 comprises observations taken at different epochs, from 2006 March to 2009 October (see Table 1 for details). HESS J0632+057 is detected 


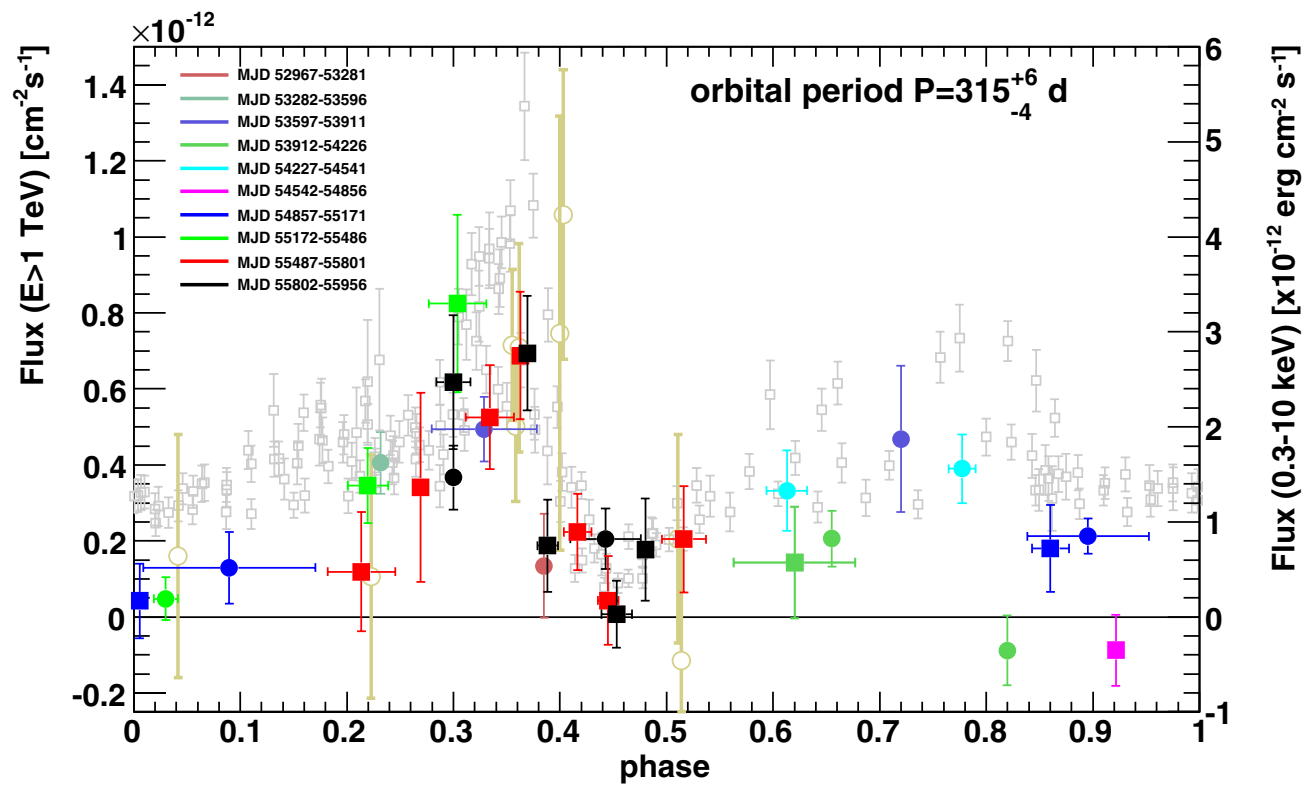

Figure 4. Integral $\gamma$-ray fluxes above $1 \mathrm{TeV}$ (vertical scale on the left) from H.E.S.S. (filled round markers), MAGIC (brown round open markers, scaled to $1 \mathrm{TeV}$ assuming a power law with index -2.6; Aleksić et al. 2012) and VERITAS (filled squared markers). X-ray fluxes (0.3-10 keV) are shown as measured by Swift-XRT (open square gray marker; vertical scale on the right). All measurements are folded with the orbital period of 315 days; the colors indicate different orbits. Vertical error bars show $1 \sigma$ statistical uncertainties; horizontal error bars indicate the width of the corresponding observing interval.

(A color version of this figure is available in the online journal.)
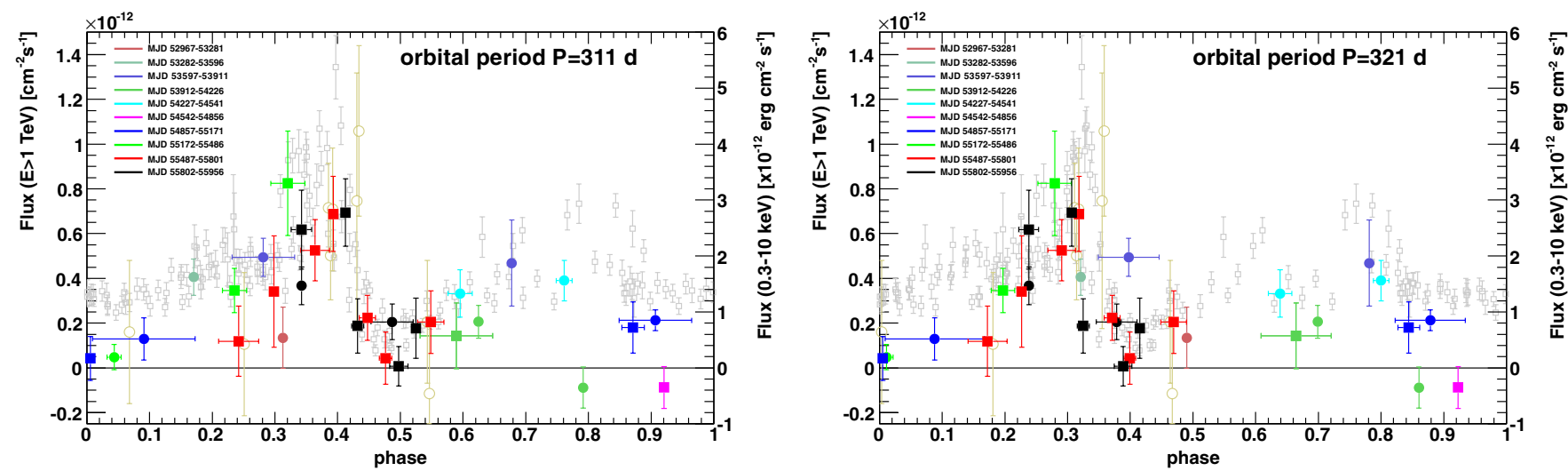

Figure 5. Impact of the $1 \sigma$ error of the orbital phase on the phase-folded light curves. Phase-folded light curve using an orbital period of 311 (left) and 321 (right) days. The maximum of the $\mathrm{X}$-ray light-curve coincides with that at $\mathrm{TeV}$ energies taking both values for the period and occurs at orbital phases $\sim 0.3$. Enhanced $\mathrm{X}$-ray emission at phases $\sim 0.6-0.9$ is also observed in the two panels, for which most of the corresponding $\mathrm{TeV}$ data points leading to the source detection in this phase interval are still included. See Figure 4 for a description of the figure axes, markers, and error bars.

(A color version of this figure is available in the online journal.)

at a significance level of $7.7 \sigma$ when all data falling in this phase interval are considered. The search for $\mathrm{TeV}$ emission has been performed in this phase range as defined by the presence of a second/smaller X-ray high-state. Therefore, no trial factor penalty needs to be applied to the significance estimation. ${ }^{69}$

\footnotetext{
${ }^{69}$ An upper limit on the number of trials for a blind search of significant emission in the phase-folded light curve can nevertheless be derived as follows: The light curve is first divided into 10 bins, and a significant detection is then evaluated for all intervals of $0.1,0.2,0.3, \ldots, 0.9$ and 1.0 width in orbital-phase, without repetition (e.g., intervals of 0.3 width, one has to consider the cases $0.0-0.1+0.1-0.2+0.2-0.3,0.0-0.1+0.1-0.2+$ $0.3-0.4, \ldots, 0.7-0.8+0.8-0.9+0.9-1.0$.$) In each case, a number of trials$ $=10 ! /[n !(10-n) !]$ is obtained, where $n=1,2,3, \ldots, 9$. The total number of trials resulting from this computation is 1275 . A $7.7 \sigma$ detection would be reduced to $\sim 6.7 \sigma$ in this extreme case using $P_{\mathrm{t}}=1.0-(1.0-P)^{N_{\text {trials }}}\left(P_{\mathrm{t}}\right.$ and $P$ are the pre- and post-trial probabilities, respectively, and $N_{\text {trials }}$ is the number of trials), which is still highly significant. Note that this is a true lower limit on the detection significance, as we do not require continuity when merging different phase intervals, which would further reduce the total number of trials.
}

Moreover, the variability index for the 18 VHE flux points outside of the phase bins around the main maximum (phases $0.2-0.4$ ) has been computed. This calculation yields a value for the variability index of $V=52.3$, corresponding to a probability of $2 \times 10^{-5}$, suggesting that there may exist variations in the source VHE light curve away from the main peak. A likelihood ratio test was also performed to further explore if the detection in phases $0.6-0.9$ constitutes a significant secondary maximum above the baseline level. This baseline flux is computed by adding all data in the phase ranges $0.0-0.2,0.4-0.6$, and $0.9-1.0$. The likelihood function is then defined as a product of two Gaussian distributions of flux measurements $\phi_{0.6-0.9}$ and $\phi_{\text {base }}$ for the emission in phases $0.6-0.9$ and in the baseline range, respectively, stating that $\phi_{0.6-0.9}$ is a factor $K_{0.6-0.9}$ times higher than $\phi_{\text {base }}$. The likelihood-ratio test provides a value for $K_{0.6-0.9}$ in the range $[0.83,3.90]$ at a $99.7 \%(3 \sigma)$ confidence level, with a best fit value $K_{0.6-0.9}=1.72$. Therefore, although the emission 

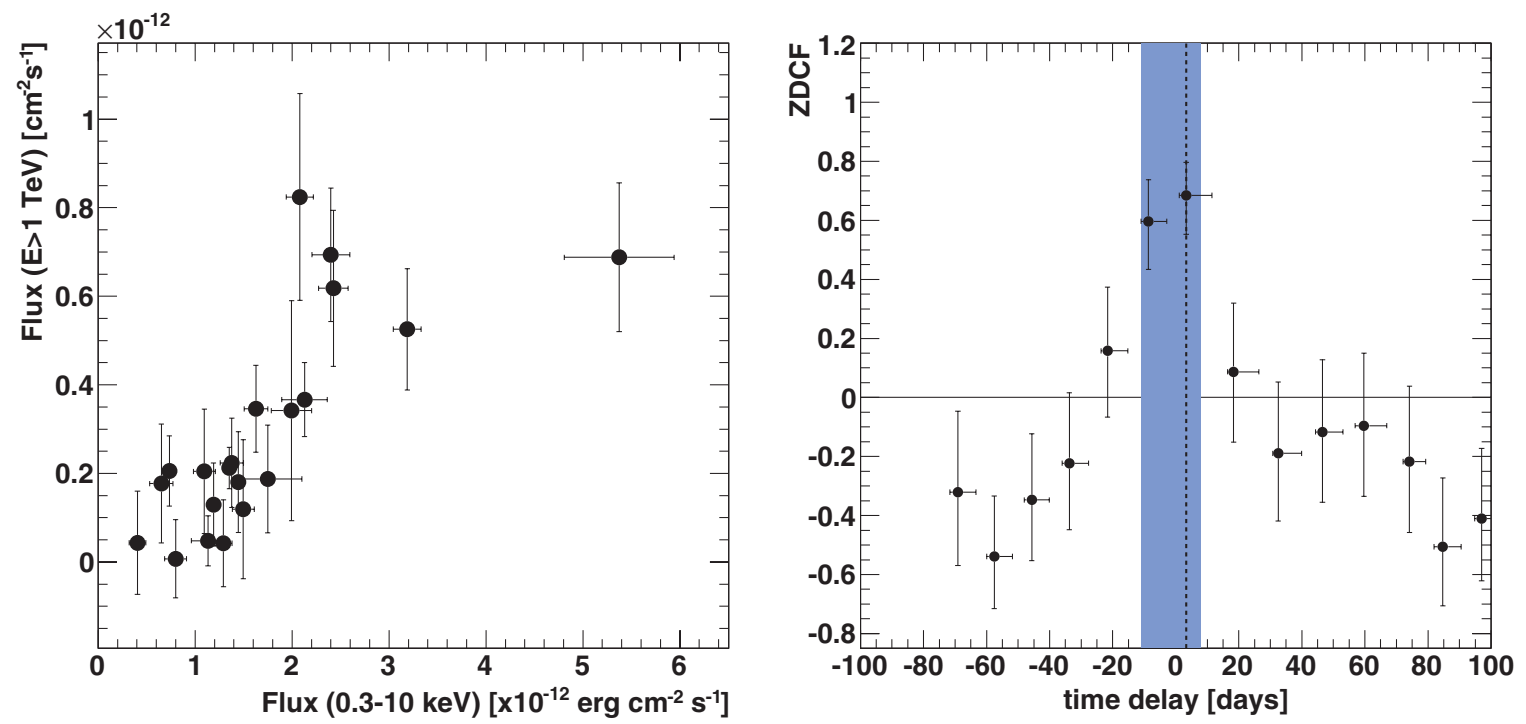

Figure 6. Left: integral $\gamma$-ray fluxes $(>1 \mathrm{TeV})$ vs. X-ray $(0.3-10 \mathrm{keV})$ fluxes for contemporaneous VHE/X-ray observations. X-ray data were selected in a \pm 2.5 day interval centered on the VHE observing dates. Right: Z-transformed discrete correlation (Z-DCF) between the Swift-XRT light curve and the combined VERITAS and H.E.S.S. gamma-ray data. The error bars denote the $1 \sigma$ sampling errors resulting from a MC-based error calculation as described in the text. The dashed line and the blue band indicate the most likely time lag between X-ray and VHE data of $3.3_{-10.8}^{+8.1}$ days and the corresponding $68 \%$ fiducial interval.

(A color version of this figure is available in the online journal.)

Table 3

Outcome of the Spectral Analysis of VHE Photons

\begin{tabular}{lcccc}
\hline \hline Year & $\begin{array}{c}\text { Orbital } \\
\text { Phase }\end{array}$ & $\begin{array}{c}\text { Flux Normalization Constant }\left(\times 10^{-13}\right) \\
\text { at } 1 \mathrm{TeV}\left(\mathrm{cm}^{-2} \mathrm{~s}^{-1} \mathrm{TeV}^{-1}\right)\end{array}$ & Photon Index & $\chi^{2} / N$ \\
\hline VERITAS 2010 & $0.2-0.4$ & $(6.4 \pm 1.0)$ & $2.2 \pm 0.4$ & $1.7 / 3$ \\
VERITAS 2011 & $0.2-0.4$ & $(11.0 \pm 1.1)$ & $2.5 \pm 0.2$ & $4.2 / 4$ \\
VERITAS 2012 & $0.2-0.4$ & $(6.4 \pm 0.8)$ & $2.3 \pm 0.2$ & $5.8 / 6$ \\
\hline H.E.S.S. 2004-2012 & $0.2-0.4$ & $(5.7 \pm 0.7)$ & $2.3 \pm 0.2$ & $32.0 / 31$ \\
VERITAS 2010-2012 & $0.2-0.4$ & $(7.7 \pm 0.5)$ & $2.6 \pm 0.1$ & $6.0 / 6$ \\
H.E.S.S. 2004-2012 & $0.6-0.9$ & $(3.9 \pm 0.7)$ & $2.4 \pm 0.2$ & $44.0 / 27$ \\
\hline H.E.S.S. 2004/2005 & & $(9.1 \pm 1.7)$ & $2.53 \pm 0.6$ & $\ldots$ \\
MAGIC 2012 & & $(12 \pm 0.3)$ & $2.6 \pm 0.3$ & $\cdots$ \\
\hline
\end{tabular}

Notes. The table lists the results of the power-law fits to the differential energy spectra; see Figure 7. The H.E.S.S. and MAGIC results from the literature are taken over a phase range $0.2-0.5$, but with different observational coverage of the light curve. Errors are $1 \sigma$ statistical errors only. The systematic error on the flux constant is typically $20 \%$ and on the spectral index $\approx 0.1$.

a Aharonian et al. (2007).

b Aleksić et al. (2012).

at orbital phases $0.6-0.9$ is higher than the baseline flux, it cannot be claimed as a secondary peak with the present data set at a high confidence level.

The correlation between gamma-ray and X-ray fluxes for 21 roughly contemporaneous observations is shown in Figure 6. $\mathrm{X}$-ray data were selected within a \pm 2.5 day interval around the VHE observing dates. Emission in these energy bands is significantly correlated $\left(\mathrm{ZDCF} / \mathrm{ZDCF}_{\text {error }}=5.6\right.$ at a time lag of zero). The time lag between gamma-ray and $\mathrm{X}$-ray data is consistent with zero $\left(\tau_{\mathrm{ZDCF}}=+3.3_{-10.8}^{+8.1}\right.$ days).

In Figure 7 the differential energy spectra are shown for gamma-ray energies above $200 \mathrm{GeV}$ during the high-flux phases $0.2-0.4$ and at phases $0.6-0.9$. Figure 8 shows the broad-band $\mathrm{SED}$ from X-ray to $\mathrm{TeV}$ energies. The shapes of the individual VHE spectra are consistent with a power-law distribution; see Table 3 for further details.

The differential energy spectrum at VHEs has been measured for three different orbits (2010, 2011 and 2012) and no significant variability in photon index or flux normalization is observed. The spectral results are in agreement with those reported in Aharonian et al. (2007) and Aleksić et al. (2012). The H.E.S.S. and VERITAS measurements presented here are fully compatible within statistical and systematic uncertainties. It should be noted that while the phase ranges for these spectral analyses are similar, the coverage by observations inside this phase range is very different for different observation campaigns.

\section{DISCUSSION}

The long-term X-ray and $\mathrm{TeV}$ observations of HESS J0632+057 at X-ray energies reported here allow for the first time the modulation of the source gamma-ray flux to be characterized in a wide orbital phase range, making use of a refined value of the orbital period of the system derived from an updated X-ray data set. Below, the implications of the results are briefly discussed, focusing on the phase-folded $\mathrm{X}$-ray and $\mathrm{TeV}$ light curves and on the correlation of the emission observed at 

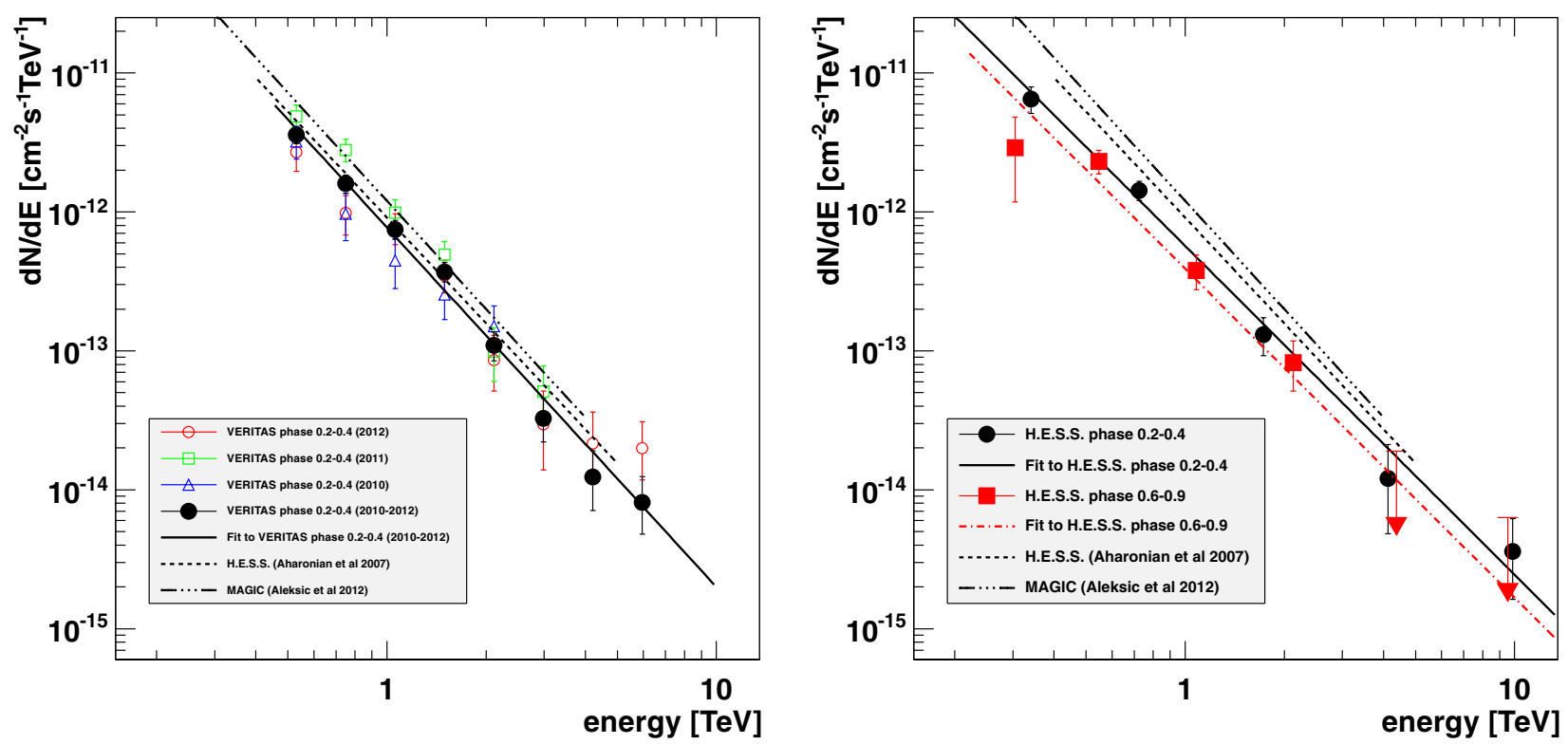

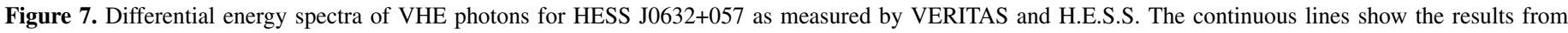

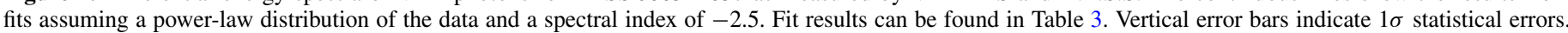

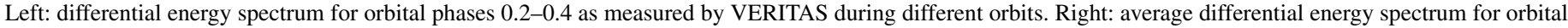

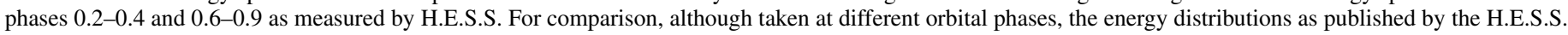

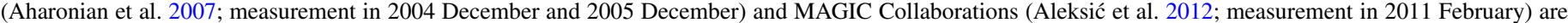
indicated by the dashed and dot-dashed lines, respectively.

(A color version of this figure is available in the online journal.)

both energy bands. The findings are put in the context of current scenarios proposed to explain the high-energy emission in gamma-ray binaries, and compared to the results obtained for other similar systems.

\subsection{On the X-Ray/TeV Phase-Folded Light Curves of HESS J0632+057}

The X-ray light curve of HESS J0632+057 shows two distinct periods of enhanced emission (see Figure 3 ). The first one, sharper and higher, appears at orbital phases $\sim 0.3$, which corresponds to about 100 days after periastron passage (Casares et al. 2012). The second one is found at phases between $\sim 0.6$ and 0.9. It appears to be of broader profile with a lower flux peak, although it is more irregularly sampled in the Swift-XRT data set (the orbital period $\sim 315$ days and the source position with respect to the Sun made Swift unable to cover this phaserange in detail during the previous few cycles). At VHEs, the source has been repeatedly detected at orbital phases $\sim 0.3$ with the VERITAS, H.E.S.S., and MAGIC observatories. In addition, the analysis of the H.E.S.S. data at orbital phases in the range 0.6-0.9 reported here (see Section 3) has yielded a detection of the source at $\mathrm{TeV}$ energies for the first time in this phase range, in rough coincidence with the secondary bump observed at $\mathrm{X}$-rays. However, only a few data points characterize the emission at these orbital phases.

A double X-ray peak pattern has been observed in other gamma-ray binaries. In LS I +61 303, a sharp X-ray peak arises at orbital phases $\sim 0.6$ (periastron is at phase $\sim 0.2$ ), whilst a broader second peak at orbital phases in the range $0.8-1.0$ has been reported, e.g., in Anderhub et al. (2009; see however Li et al. 2011, 2012 and Chernyakova et al. 2012 for a study on the long-term evolution of the phase-folded X-ray light curve of the source). A similar behavior is seen also in the X-ray light curve of the newly discovered system 1FGL J1018.6-5856 (Fermi-LAT Collaboration 2012). In this case, however, the orbital parameters are still lacking, and a correspondence of the position of the two peaks in the phase-folded light curve and the relative orientation of the compact object and the companion star has not been derived yet. For the well-studied system LS 5039, which contains an O-type companion star as in 1FGL J1018.6-5856, both the X-ray and $\mathrm{TeV}$ maxima are produced close to the system inferior conjunction, when the compact object is in front of the star, with no distinct double-peak structure in the X-ray light curve (Takahashi et al. 2009; Hofmann et al. 2009). In the case of PSR B1259-63, composed of a pulsar and a Be companion star, enhanced $\mathrm{X}$-ray and $\mathrm{TeV}$ emission is found close to periastron. A double X-ray peak has been observed at these orbital phases, but in this case they have been interpreted as the compact object twice crossing the companion's circumstellar disk (Chernyakova et al. 2009). No double-peak structure has been claimed yet at $\mathrm{TeV}$ energies in the data collected in the 2004, 2007 and 2011 periastron passages (H.E.S.S. Collaboration 2013). Finally, we note that the peak and dip structure in the X-ray light curve of HESS J0632+057 resembles that observed in Eta Carinae, which is thought to be due to the strong interaction of stellar winds as well as to the geometrical properties of the system orbit (Corcoran 2005). However, most of the X-ray flux cannot arise from the shocked stellar wind as the resulting thermal spectrum does not fit well the observed hard X-ray spectrum (Falcone et al. 2010).

The VHE emission observed in known compact gamma-ray binaries can be strongly modulated along the binary orbit due to gamma-ray absorption in the photon field of the companion star (see, e.g., Böttcher \& Dermer 2005; Dubus 2006b, and references therein). In addition, in leptonic models a relatively high target photon field density is required for gamma-rays to be produced through inverse Compton (IC) emission processes, whilst the anisotropy of the companion star photon field further introduces a phase dependence of the spectrum of the upscattered photons (see, e.g., Jackson 1972; Dubus et al. 2010). The detection of VHE emission is therefore subject to the balance 


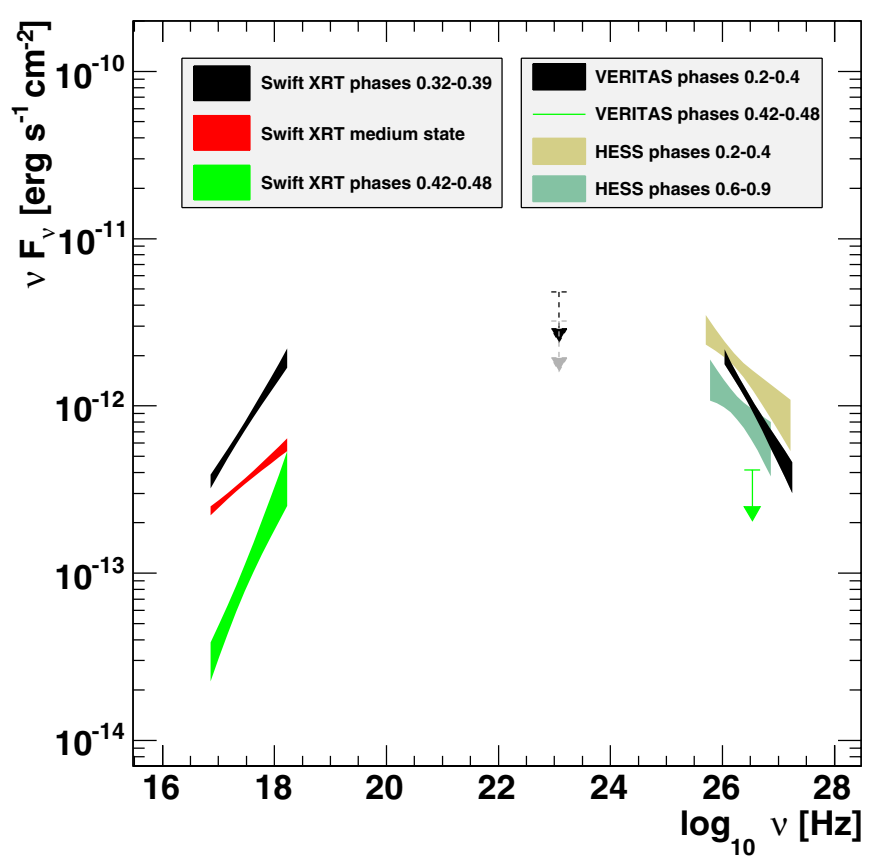

Figure 8. Spectral energy distribution for HESS J0632+057 as measured by VERITAS, H.E.S.S., Swift-XRT, and Fermi-LAT (Caliandro et al. 2013). The downward pointing arrow at VHE is an upper flux limit at $95 \%$ confidence level for phases $0.42-0.48$. Details for the high, medium, and low state X-ray measurements are given in Table 2. The integral upper limit above $100 \mathrm{MeV}$ measured by Fermi-LAT (confidence level 95\%) has been converted to a differential flux value assuming a spectral index $\Gamma=-2$. (black dashed arrow) and $\Gamma=-2.5$ (gray dashed arrow) and is shown at an energy of $500 \mathrm{MeV}$. The butterfly plots indicate the $1 \sigma$ statistical errors of the power-law fit to the data points.

(A color version of this figure is available in the online journal.)

between the intrinsic gamma-ray flux and the attenuation factor, which will depend on the system geometry and therefore on the orbital phase. Considering the orbital parameters recently obtained for HESS J0632+057 (Casares et al. 2012), the VHE emission observed in the phase-folded light curve at phases $0.2-0.4$ and $0.6-0.9$ does not correspond to orbital phases in which the compact object is found close to the Be companion nor close to inferior conjunction where a low opacity to gammaray propagation is expected (note however that there are large uncertainties in the orbital solution for MWC 148, see Casares et al. 2012).

Other factors may nevertheless contribute to and even dominate the observed modulation in HESS J0632+057. In particular, fluxes may be regulated by the variability of the underlying particle population emitting at VHEs. Such variability could come from orbital-dependent adiabatic losses, which may eventually constrain the maximum energies that particles can attain, or from a discontinuous particle injection, either in a varying wind-wind shock boundary in a pulsar scenario or due to a phase-dependent accretion rate in a microquasar model.

It is worth noting that, facing a similar situation in the case of LS I +61 303, numerical simulations have shown that a shifted peak in the high-energy emission light curve may appear in an accretion-based scenario (Romero et al. 2007; see also Hayasaki \& Okazaki 2005; Orellana and Romero 2007). A shift of the TeV peak of $\sim 0.3$ orbital phases from periastron is also obtained in a pulsar scenario for the same source by Sierpowska-Bartosik \& Torres (2009; see also Zdziarski et al. 2010). For both models there is a strong dependence of the orbital modulation of the VHE emission on the geometry of the system. The orbital parameters are not known to the required level of accuracy for a more detailed comparison of the observed emission pattern with the model predictions. In addition, adiabatic losses could also be responsible for the $\mathrm{X}$-ray and $\mathrm{TeV}$ double-peak profile in the light curve of LS I + 61303 (Zabalza et al. 2011; see also Takahashi et al. 2009 in the case of LS 5039). The physical processes leading to this ad hoc adiabatic loss pattern are however not clear. If the system contains a Be star, they could be related to the structure of the stellar wind, with two distinct polar and equatorial components (see, e.g., Waters et al. 1988), or to perturbations of the Be circumstellar disk carried along or affecting orbital phases away from the closest approach during periastron passage. This would affect the emission properties in both an accretion and a pulsar-wind-based model. In the former case, the total X-ray and VHE fluxes depend linearly on the mass accretion rate, which depends in turn on the companion's wind density and on the relative velocity with respect to the compact object. If the power engine is a fast rotating pulsar, instead, the wind profile may also affect the emitter position with respect to the companion star, which would lead to different emissivities through IC upscattering of the star's photon field. The true nature of the observed modulation of the gamma-ray and X-ray light curves in HESS J0632+057 is not yet univocally determined.

\subsection{On the X-Ray/TeV Correlation in HESS J0632+057}

The results reported in Section 3 show a clear correlation between X-rays and TeV gamma-rays. Together with the observed periodicity, this correlation suggests a causal link between the emission at both energies, for instance produced by processes related to the same population of accelerated particles. The SED from X-rays to VHE gamma-rays of HESS J0632+057 (Figure 8) reveals a shape typical of non-thermal high-energy gamma-ray emitters and, in particular, resembles that of known TeV binaries, displaying hard X-ray and soft TeV spectra. Such spectral shapes can be modeled with relatively simple one-zone leptonic models, as shown, e.g., in Hinton et al. (2009) and Aleksić et al. (2012), supporting the assumption that X-rays are produced through synchrotron emission of high-energy particles which, in turn, produce the VHE emission through IC upscattering off the companion's photon field. Note however that, in contrast to other known gamma-ray binaries, HESS J0632+057 has not been detected at MeV-GeV energies, despite deep searches using $\sim 3.5 \mathrm{yr}$ of Fermi-LAT data (Caliandro et al. 2013). This could point to a similar missing correlation between $\mathrm{GeV}$ and $\mathrm{TeV}$ emission as observed in LS I + 61303 or LS 5039 (Hadasch et al. 2012).

We assume in the following that the particles dominating the emission at the $\mathrm{X}$-ray maximum at phases $\sim 0.3$ come indeed from the same population that is responsible for the $\mathrm{TeV}$ emission. We explore here the possibility that a cessation or reduction of the acceleration is the main factor responsible for the peak-to-dip transition in the X-ray light curve (phases 0.3-0.4), rather than absorption processes (Falcone et al. 2010; Bongiorno et al. 2011; see however Rea \& Torres 2011). Note that this could also imply that the X-ray emission within the dip may be dominated by a different parent particle population than that seen during the peak. In this transition, a rough characterization of the system properties can be derived. Particles will lose their energy either through radiative (mainly IC and synchrotron emission) or non-radiative processes (e.g., adiabatic expansion). In the first case, the ratio of IC over synchrotron losses as a function of the particle energy can be estimated through $r_{\mathrm{IC} / \text { sync }} \equiv f_{\mathrm{KN}} U_{\mathrm{rad}} / U_{\mathrm{mag}}$, where $U_{\mathrm{rad}}$ 
and $U_{\text {mag }}$ are the photon and magnetic field energy densities, respectively, and the factor $f_{\mathrm{KN}}$ accounts for Klein-Nishina (KN) effects in the IC cross-section (Moderski et al. 2005; see also Hinton et al. 2009). $U_{\text {rad }}$ can be estimated assuming a distance $d \sim a \approx 2.38 \mathrm{AU}$ from the emitter to the companion star ( $a$ is the semimajor axis of the orbit; Casares et al. 2012), with a radius $R_{\star}=6.0 R_{\odot}$ and a temperature $T_{\text {eff }}=27.500 \mathrm{~K}$ (Aragona et al. 2010), which yields a peak of the target photon field at $\epsilon_{0} / m_{\mathrm{e}} c^{2} \approx 1.3 \times 10^{-5}$. For $U_{\text {mag }}$, Moldón et al. (2011) reported the detection of radio emission from HESS J0632+057 observed close to the X-ray peak in 2011. The authors favor a synchrotron origin for this radio emission, and assuming equipartition of the magnetic field energy with the kinetic energy of the emitting electrons they derive a value $B \approx 20 \mathrm{mG}$. The radio and VHE emission regions could be very different in size and location, however, so they may not be characterized by the same magnetic field energy density. Assuming a range of magnetic field values $B=10,20$ and $50 \mathrm{mG}$, the ratio $r_{\mathrm{IC} / \text { sync }}=1$ would correspond to electron energies $E_{\mathrm{e}} \approx 8.7,3.4$, and $1.0 \mathrm{TeV}$, respectively. For lower and higher energies, IC and synchrotron processes would correspondingly dominate the total radiative losses. Note however that the upper limits at $\mathrm{GeV}$ energies reported in Caliandro et al. (2013), and the lack of information at hard $\mathrm{X}$-rays, prevent a more accurate evaluation of the energy output channeled through both radiation mechanisms.

Further constraints can be obtained from the X-ray phasefolded light curve. If the Swift-XRT flux has a synchrotron origin, electrons with energies $E_{\mathrm{e}} \approx 0.285\left(E_{\text {sync }} /\right.$ $5.4 \mathrm{keV})^{1 / 2}(B / 1 \mathrm{G})^{-1 / 2} \mathrm{TeV}$ are required $\left(E_{\text {sync }}\right.$ is the characteristic synchrotron energy of the emitted photon). For the range of magnetic field values $B=10,20$, and $50 \mathrm{mG}, E_{\mathrm{e}} \sim 4.5,3.2$, and $2.0 \mathrm{TeV}$, respectively, and from the considerations above, both synchrotron and IC processes would contribute significantly to the radiative particle cooling (see, e.g., Skilton et al. 2009). The total time scale for the radiative losses, $t_{\text {rad }}=\left(t_{\text {sync }}^{-1}+t_{\text {IC-KN }}^{-1}\right)^{-1}$ would range between $\sim 1.5$ days and $\sim 2$ days. This time scale is roughly comparable to that in which the X-ray flux is observed to decrease by a noticeable factor in the transition from the X-ray maximum to the X-ray dip in Figure $4\left(t_{\text {peak-dip }} \sim 0.01\right.$ phases corresponding to 3.3 days using $P=315$ days).

Regarding adiabatic losses, Moldón et al. (2011) report also on the detection of extended radio emission from HESS J0632+057 seen 30 days after the X-ray peak in 2011 January/February. ${ }^{70}$ The total extension of this emission was of the order of 50 mas, which translates to about $l_{\text {ext }} \sim 75 \mathrm{AU}$ when a distance of $\sim 1.5 \mathrm{kpc}$ to the source is assumed. If material ejected from the vicinity of the compact object expanded to reach $l_{\text {ext }}$ in $\lesssim 30$ days, an expansion velocity $v_{\exp } \gtrsim 1.2 \times 10^{8} \mathrm{~cm} \mathrm{~s}^{-1}$ would imply an adiabatic cooling time $t_{\text {ad }} \sim l_{\text {ext }} / v_{\text {exp }} \lesssim 108$ days. Therefore, radiative cooling may have dominated the total losses during the X-ray peak-to-dip transition, unless the ejected material expanded at a high velocity within the 30 day lag between the two radio observations, $v_{\text {exp }} \sim 0.25 c$, in which case $t_{\text {ad }} \approx t_{\text {rad }}$.

These crude estimates should be seen more as illustrative than as a detailed description of the true physical processes leading to the observed high-energy emission in HESS J0632+057, and alternative scenarios may be considered. On the one hand, electrons could produce X-rays through IC instead of

\footnotetext{
70 Note, however, that the flux obtained for this extended radio emission is below the rms level of that derived for the point-like source emission obtained close to the X-ray maximum.
}

synchrotron processes, reaching also the gamma-ray domain. In that case, however, the wide range of electron energies required would imply very different cooling time scales, at odds with the tight correlation observed in the $\mathrm{X}$-ray/TeV light curve. Furthermore, the lack of GeV emission (Caliandro et al. 2013), would be difficult to justify in such scenario. On the other hand, VHE emission could be produced through hadronic interactions of protons accelerated close to the compact object against ions present in the companion's wind and/or circumstellar disk. In parallel, secondary electrons/positrons would then be produced through pion decay in the same proton-proton interactions that would initiate the gamma-ray fluxes, leading to an X-ray energy flux at a level $\sim 1 / 2$ of that produced at gamma-rays (see, e.g., Kelner et al. 2006). This is not observed, however, during the $\mathrm{X}$-ray/TeV peak at phase $\sim 0.3$ (see Figure 8 ), in which a similar luminosity is radiated at both $\mathrm{X}$-rays and $\mathrm{TeV}$ energies. Note also that there is no evidence of a cutoff of the X-ray spectrum, which is relatively hard with a spectral index of $1.46 \pm 0.06$, and which may even extend into the hard X-ray domain, enhancing the total X-ray luminosity of the source.

Finally, properties of the medium like the radiation and matter density fields, rather than the intrinsic properties of the emitter, could be responsible for the simultaneous modulation that shapes the observed fluxes at X-ray and gamma-ray energies in a periodic way. However, it should be noted that VHE radiation is affected mainly by the interaction with the companion star photon field. Conversely, X-ray fluxes are mainly reprocessed through interactions with the ambient matter. In this regard, an enhancement of the local matter density might be expected during the X-ray light curve minima, which has not been observed (see, e.g., Bongiorno et al. 2011; Rea \& Torres 2011). This, together with the tight $\mathrm{X}-\mathrm{ray} / \mathrm{TeV}$ correlation implying a similar modulation of the fluxes at both energy bands, favors a scenario in which the variability arises from a modulation of a common underlying emitting-particle population.

A correlation of X-ray and $\mathrm{TeV}$ emission has also been observed in other gamma-ray binaries. In particular, correlated $\mathrm{X}$-ray/VHE emission has been reported in the case of LS I +61 303 through simultaneous MAGIC, XMM-Newton, and SwiftXRT observations in a multiwavelength campaign conducted in 2007 (Anderhub et al. 2009). The correlation result was not apparent however in later observations of the source (Acciari et al. 2011), and a change in the source/medium properties has also been observed in recent gamma-ray observations (Acciari et al. 2011; Aleksić et al. 2012; Hadasch et al. 2012), which display strong deviations of the source phase-folded flux profiles as compared to older data. The processes leading to such transitions in the modulation of the light curve are not clear, although a relation to the superorbital variability of the companion star seen at lower radio and X-ray energies has been suggested (Gregory 2002; see also Li et al. 2012; Chernyakova et al. 2012). As compared to LS I +61 303, the emission from HESS J0632+057 seems to be steadier, with a remarkably lower orbit-to-orbit variability and only small deviations from the main, long-term pattern observed for more than five years. Further observations are required to assess whether a superorbital modulation is also present in HESS J0632+057.

\section{FINAL REMARKS}

HESS J0632+057 together with LS I +61 303 and PSR B1259-63 is one of the three gamma-ray binaries known to contain a Be companion star. Common processes leading to the 
production of non-thermal emission from radio to VHE gamma rays may explain the broad-band energy distribution in all of them. However, differences in their orbital parameters and the nature of the still-unknown power sources in LS I + 61303 and HESS J0632+057, may ultimately define their individual observational properties, including the phase-folded patterns observed in each case. Detailed models with a realistic description of the geometry of the orbit, the interaction of the stellar wind with the wind or jet of the compact object, and the distribution of photon and matter fields, are necessary to get a deeper understanding of the system and its orbital variability.

Due to their variable and relatively well-constrained environment, the characterization of the high-energy behavior of gamma-ray binaries has become an important research field in recent years. New candidates have been discovered (e.g., 1FGL J1018.6-5856; Fermi-LAT Collaboration 2012; Abramowski et al. 2012) and unexpected features are being observed in some of the known sources as, e.g., in PSR B1259-63 (Abdo et al. 2011) and LS I +61 303 (Acciari et al. 2011). Furthermore, detailed numerical simulations are being run (e.g., Romero et al. 2007; Sierpowska-Bartosik \& Torres 2008; Perucho et al. 2010; Perucho \& Bosch-Ramon 2012; Bosch-Ramon et al. 2012) as well as new scenarios are being proposed to explain them (see, e.g., Khangulyan et al. 2012; see also Torres et al. 2012; Zabalza et al. 2013; Bednarek \& Sitarek 2013). These and future studies, together with the improved capabilities of next-generation VHE observatories, may provide new clues to unveil the physics behind gamma-ray binaries.

This research is supported by grants from the U.S. Department of Energy Office of Science, the U.S. National Science Foundation and the Smithsonian Institution, by NSERC in Canada, by Science Foundation Ireland (SFI 10/RFP/AST2748) and by STFC in the U.K. We acknowledge the excellent work of the technical support staff at the Fred Lawrence Whipple Observatory and at the collaborating institutions in the construction and operation of the instrument. G.M. acknowledges support through the Young Investigators Program of the Helmholtz Association. M.D. is funded by contract ERC-StG-259391 from the European Community.

The support of the Namibian authorities and of the University of Namibia in facilitating the construction and operation of H.E.S.S. is gratefully acknowledged, as is the support by the German Ministry for Education and Research (BMBF), the Max Planck Society, the French Ministry for Research, the CNRSIN2P3 and the Astroparticle Interdisciplinary Programme of the CNRS, the U.K. Particle Physics and Astronomy Research Council (PPARC), the IPNP of the Charles University, the South African Department of Science and Technology and National Research Foundation, and by the University of Namibia. We appreciate the excellent work of the technical support staff in Berlin, Durham, Hamburg, Heidelberg, Palaiseau, Paris, Saclay, and in Namibia in the construction and operation of the H.E.S.S. equipment.

Facilities: VERITAS, HESS, Swift

\section{REFERENCES}

Abdo, A. A., Ackermann, M., Ajello, M., et al. (Fermi-LAT Collaboration) 2010, ApJS, 188, 405

Abdo, A. A., Ackermann, M., Ajello, M., et al. (Fermi-LAT Collaboration) 2011, ApJ, 736, 11

Abramowski, A., Acero, F., Aharonian, F., et al. (H.E.S.S. Collaboration) 2012, A\&A, 541A, 5
Acciari, V. A., Aliu, E., Arlen, T., et al. (VERITAS Collaboration) 2009, ApJL, 698, L94

Acciari, V. A., Aliu, E., Arlen, T., et al. (VERITAS Collaboration) 2011, ApJ, 738, 3

Acciari, V. A., Beilicke, M., Blaylock, G., et al. (VERITAS Collaboration) 2008, ApJ, 679, 1427

Aharonian, F. A., Akhperjanian, A. G., Aye, K.-M., et al. (H.E.S.S. Collaboration) 2005a, A\&A, 442, 1

Aharonian, F. A., Akhperjanian, A. G., Aye, K.-M., et al. (H.E.S.S. Collaboration) 2005b, Sci, 309, 746

Aharonian, F. A., Akhperjanian, A. G., Aye, K.-M., et al. (H.E.S.S. Collaboration) 2006, A\&A, 457, 899

Aharonian, F. A., Akhperjanian, A. G., Bazer-Bachi, A. R., et al. (H.E.S.S. Collaboration) 2007, A\&A, 469, L1

Albert, J., Aliu, E., Anderhub, H., et al. (MAGIC Collaboration) 2006, Sci, 312, 1771

Albert, J., Aliu, E., Anderhub, H., et al. (MAGIC Collaboration) 2007, ApJ, 665,51

Albert, J., Aliu, E., Anderhub, H., et al. (MAGIC Collaboration) 2009, ApJ, 693,303

Aleksić, J., Alvarez, E. A., Antonelli, L. A., et al. (MAGIC Collaboration) 2012, ApJL, 754, L10

Alexander, T. 1997, in Astronomical Time Series, ed. D. Maoz, A. Sternberg, \& E. M. Leibowitz (Dordrecht: Kluwer), 163

Anderhub, H., Antonelli, L. A., Antoranz, P., et al. (MAGIC Collaboration) 2009, ApJL, 706, L27

Aragona, C., McSwain, M. V., \& De Becker, M. 2010, ApJ, 724, 306

Arnaud, K. A. 1996, in ASP Conf. Ser. 101, Astronomical Data Analysis Software and Systems V, ed. G. Jacoby \& J. Barnes (San Francisco, CA: ASP), 17

Ball, L., \& Kirk, J. G. 2000, APh, 12, 335

Bednarek, W., \& Sitarek, J. 2013, MNRAS, 430, 2951

Berge, D., Funk, S., \& Hinton, J. 2007, A\&A, 466, 1219

Blackburn, J. K. 1995, in ASP Conf. Ser. 77, Astronomical Data Analysis Software and Systems IV, ed. R. A. Shaw, H. E. Payne, \& J. J. E. Hayes (San Francisco, CA: ASP), 367

Bogovalov, S., Khangulyan, D., Koldoba, A. V., et al. 2008, MNRAS, 387, 63B

Bongiorno, S. D., Falcone, A. D., Stroh, M., et al. 2011, ApJL, 737, L11

Bordas, P. (H.E.S.S. Collaboration), \& Maier, G. (VERITAS Collaboration) 2012, in AIP Conf. Proc. 1505, High Eenergy Gamma-Ray Astronomy: 5th International Meeting on High Energy Gamma-Ray Astronomy, ed. F. A. Aharonian, W. Hofman, \& F. M. Rieger (Melville, NY: AIP), 366

Bosch-Ramon, V., Barkov, M. V., Khangulyan, D., \& Perucho, M. 2012, A\&A, 544, 59

Böttcher, M., \& Dermer, C. D. 2005, ApJ, 634, 81

Burrows, D. N., Hill, J. E., Nousek, J. A., et al. 2005, SSRv, 120, 165

Caliandro, G. A., Hill, A. B., Torres, D. F., et al. 2013, MNRAS, 436, 740

Casares, J., Ribó, M., Ribas, I., et al. 2012, MNRAS, 421, 1103

Chernyakova, M., Neronov, A., Aharonian, F. A., et al. 2009, MNRAS, 397 212

Chernyakova, M., Neronov, A., Molkov, S., et al. 2012, ApJL, 747, L29

Corcoran, M. F. 2005, AJ, 129, 2018

de Naurois, M., \& Rolland, L. 2009, APh, 32, 231

Dubus, G. 2006a, A\&A, 456, 801

Dubus, G. 2006b, A\&A, 451, 9

Dubus, G. 2013, A\&ARv, 21, 64

Dubus, G., Cerutti, B., \& Henri, G. 2010, A\&A, 516, A18

Edelson, R. A., \& Krolik, J. H. 1988, ApJ, 333, 646

Falcone, A., Grube, J., Hinton, J., et al. 2010, ApJL, 708, L52

Fermi-LAT Collaboration 2012, Sci, 335, 189

Gregory, P. C. 2002, ApJ, 575, 427

Hadasch, D., Torres, D. F., Tanaka, T., et al. 2012, ApJ, 749, 54

Hartman, R. C., Bertsch, D. L., Bloom, S. D., et al. 2012, ApJS, 123, 79

Hayasaki, K., \& Okazaki, A. T. 2005, MNRAS, 360, L15

H.E.S.S. Collaboration 2013, A\&A, 551, A94

Hillas, M. 1985, in Proc. 19th ICRC, ed. F. C. Jones, Vol. 3 (La Jolla, CA: NASA), 445

Hinton, J., Skilton, J. L., Funk, S., et al. 2009, ApJL, 690, L101

Hoffmann, A. D., Klochkov, D., Santangelo, A., et al. 2009, A\&A, 494, L37

Hog, E., Kuzmin, A., Bastian, U., et al. 1998, A\&A, 335, L65

Jackson, J. C. 1972, NPhS, 236, 39

Kelner, S. R., Aharonian, F. A., \& Bugayov, V. V. 2006, PhRvD, 74, 034018

Khangulyan, D., Aharonian, F. A., Bogovalov, S. V., et al. 2012, ApJ, 752, 17

Krawczynski, H., Carter-Lewis, D. A., Duke, C., et al. 2006, APh, 25, 380

Li, J., Torres, D. F., Zhang, S., et al. 2011, ApJ, 733, 89

Li, J., Torres, D. F., Zhang, S., et al. 2012, ApJL, 744, L13

Li, T. P., \& Ma, Y. Q. 1983, ApJ, 272, 317 
Maier, G. 2011, in Proc. 32nd ICRC (Beijing: IUPAP) Maraschi, L., \& Treves, A. 1981, MNRAS, 194, 1

Mirabel, I. 2012, Sci, 335, 175

Mirabel, I. F., \& Rodriguez, L. F. 1994, Natur, 371, 46

Moderski, R., Sikora, M., Coppi, P. S., \& Aharonian, F. A. 2005, MNRAS, 363,954

Moldón, J., Ribó, M., \& Paredes, J. M. 2011, A\&A, 553, L7

Moldón, J., Ribó, M., \& Paredes, J. M. 2012, A\&A, 548, 103

Morgan, W. W., Code, A. D., \& Whitford, A. A. 1955, ApJS, 2, 41

Morrison, R., \& McCammon, D. 1983, ApJ, 270, 119

Orellana, M., \& Romero, G. E. 2007, Ap\&SS, 309, 333

Perucho, M., \& Bosch-Ramon, V. 2012, A\&A, 539, A57

Perucho, M., Bosch-Ramon, V., \& Khangulyan, D. 2010, A\&A, 512, 4

Rea, N., \& Torres, D. 2011, ApJL, 7373, L12

Rolke, W., \& Lopez, A. 2001, NIMA, 458, 745
Romero, G., Okazaki, A. T., Orellana, M., \& Owocki, S. P. 2007, A\&A, 474,15

Sierpowska-Bartosik, A., \& Torres, D. 2008, APh, 30, 239

Sierpowska-Bartosik, A., \& Torres, D. 2009, ApJ, 693, 1462

Skilton, J., Pandey-Pommier, M., Hinton, J., et al. 2009, MNRAS, 399, 317

Takahashi, T., Kishishita, T., Uchiyama, Y., et al. 2009, ApJ, 697, 592

Taylor, A. R., \& Gregory, P. C. 1984, ApJ, 283, 273

Torres, D. F., Rea, N., Esposito, P., et al. 2012, ApJ, 744, 106

van Soelen, B., \& Meintjes, P. J. 2011, MNRAS, 412, 1721

Waters, L. B. F. M., Taylor, A. R., van den Heuvel, E. P. J., et al. 1988, A\&A 198,200

Zabalza, V., Bosch-Ramon, V., Aharonian, F., \& Khangulyan, D. 2013, A\&A, 551,17

Zabalza, V., Paredes, J. M., \& Bosch-Ramon, V. 2011, A\&A, 527, A9

Zdziarski, A. A., Neronov, A., \& Chernyakova, M. 2010, MNRAS, 403, 1873 